
\title{
28 Research Square \\ Community and Transcriptome Foliar Mycobiota Transitions in Response to Pathogenic Conifer Needle Interactions
}

Jessa Ata

Colorado State University

Jorge Caballero

Colorado State University

Zaid Abdo

Colorado State University

Stephen Mondo

Joint Genome Institute

Jane Stewart ( $\boldsymbol{\sim}$ jane.stewart@colostate.edu )

Colorado State University

\section{Research Article}

Keywords: Pathogenicity factors, biotrophic, upregulated genes, dysbiosis, foliar pathogen, plant defense

Posted Date: February 4th, 2022

DOI: https://doi.org/10.21203/rs.3.rs-1258752/v1

License: (c) (i) This work is licensed under a Creative Commons Attribution 4.0 International License. Read Full License 


\section{Abstract}

Profiling the host-mycobiota interactions in healthy vs. diseased forest ecosystems helps understand the dynamics of understudied yet increasingly important threats to forest health that are emerging due to climate change. We analyzed the structural and functional changes of the mycobiota and the responses of Pinus contorta in the Lophodermella needle cast pathosystem through metabarcoding and metatranscriptomics. When needles transitioned from asymptomatic to symptomatic, dysbiosis of the mycobiota occurred, but with an enrichment of Lophodermella pathogens. Many pathogenicity-related genes were highly expressed by the mycobiota at the necrotrophic phase, suggesting an active pathogen response that are absent in asymptomatic needles. This study also revealed that Lophodermella spp. are members of a healthy needle mycobiota that have a latent lifestyles suggesting that other pine needle pathogens may have similar biology. Interestingly, $P$. contorta upregulated defense genes in healthy needles, indicating response to fungal recognition, while a variety of biotic and abiotic stresses genes were activated in diseased needles. Further investigation to elucidate the possible antagonistic interplay of other biotic members leading to disease progression and/or suppression is warranted. This study provides insights into microbial interactions in non-model pathosystems and contributes to the development of new forest management strategies against emerging latent pathogens.

\section{Introduction}

Endophytes are microorganisms that colonize plant tissues without causing symptoms and are known to play a vital role in plant health [1]. The wide species diversity of endophytes has been associated with a suite of diverse, but often unknown or poorly understood, ecological functions [2]. In many host-endophyte interactions, their symbiosis with host plants provides beneficial effects on host plant fitness and survival amid stressors, particularly pests and pathogens [3]. Directly or indirectly, effects against pests and pathogens occur through improving plant physiology, hyperparasitism, production of secondary metabolites, etc. $[4,5]$. On the other hand, endophytes can also be harmful latent pathogens that remain dormant until favorable environmental conditions occur or when hosts become weakened when under stress [6, 7]. Some fungal endophytes can also increase disease severity by enabling pathogen infections $[8,9]$. These relationships, however, are only part of a complex continuum of host-endophyte interactions that have a significant impact on forest ecosystem health.

These diverse ecological roles of the microbiota can trigger different plant responses to microbial infection and invasion. While similar initial defense responses could be elicited by both pathogenic and non-pathogenic endophytes, some symbiotic microbes have sophisticated systems recognized by the host plant which result in the downregulation of defense genes [10]. Similarly, in a direct fungal-host interaction, [11] demonstrated that unlike for endophytes, plants recognize pathogens via overproduction of host defense enzymes. These differing host responses by the host plant to pathogenic and endophytic fungal infections could likely indicate plant evolutionary adaptations that emerged through pathogenic interactions [12].

Profiling the mycobiota in a pathosystem and their interaction with the host plant can improve our understanding of disease development and suppression. Shifts in the microbial diversity $[13,14]$ and gene expression of the microbiome and host in healthy versus diseased plant systems were demonstrated to have important structural and functional impacts on disease development $[15,16]$. This is particularly relevant as our view of disease development shifts from the classical "one-microbe-one disease" to a more complex nature that involves coinfection of a concert of microbial organisms interacting with their environment, or a pathobiome $[13,17,18]$. Conversely, disease suppressive activities of endophytic microbial consortia have been continuously explored to reduce disease impacts [19].

Page 2/29 
Lophodermella concolor (Dearn.) Darker and L. montivaga Petrak of Rhytismataceae are potentially obligate pathogens causing needle cast on Pinus contorta [20], which is naturally distributed along the western region of northern America. Disease symptoms on infected hosts include needle discoloration and defoliation which could negatively impact growth when severe [20]. Recently, two epidemics caused by these two pathogens were recorded in Colorado, USA [21]. Though found on the same host and in the same sites in Colorado, their ecological interaction on an individual host has not been well-documented. It has been reported that, among the infected sites, all but one had only a single pathogen occurrence based on hysterothecia development, which may be due to unknown ecological differences between the two species [21] or ecological competition prevailed by the most dominant and/or aggressive pathogen [22]. Additionally, little information is known about the interaction between these pathogens and other fungal endophytes in P. contorta. However, invasion of other fungi was reported to inhibit ascocarp development [23].

This study aims to understand the interaction between the needle mycobiota with L. concolorand with L. montivaga in $P$. contorta. Using next generation sequencing, we specifically examined the fungal endophytic community composition and gene expression in asymptomatic and symptomatic needles of $P$. contorta trees infected with Lophodermella pathogens. We also explored the differences in plant responses at asymptomatic and symptomatic states. We expected a unique fungal assemblage in asymptomatic needles that suppresses disease development compared to symptomatic needles. In symptomatic needles, the pathogenicity-related mechanisms of $L$. concolor or L. montivaga provide a competitive advantage either through niche competition or antagonism over other fungal endophytes lowering fungal diversity. Based on previously observed morphological and ecological differences between the two pathogens, we further hypothesized that variations exist in the gene expression profiles between $L$. concolor and L. montivaga and in their interaction within the host. Our findings partially confirm these hypotheses, and elucidate the interaction among the needle mycobiome and plant host in the transition from healthy to diseased state.

\section{Materials And Methods}

\section{Sample collection and preparation}

Second-year asymptomatic and symptomatic needles of $P$. contorta were randomly collected at breast height (approx. $1.37 \mathrm{~m}$ ) from 60 trees infected with either L. concolor or L. montivaga from nine sites in Gunnison National Forest in June 2018 and 2019 (Table 1). Symptomatic needles were brown or discolored with hysterothecia of either $L$.

concolor or L. montivaga, while asymptomatic needles were green. From each tree, two to three needles from different fascicles (approx. $0.1 \mathrm{~g}$ in total) were pooled together as either symptomatic $(n=60)$ or asymptomatic $(n=60)$ needle samples. To remove superficial contamination, needles were washed with $0.2 \%$ Tween solution and vortexed at minimum speed for 10 mins. Samples were then rinsed in $70 \%$ ethanol for one min and dried before storing in $-20^{\circ} \mathrm{C}$ $[24,25]$.

To evaluate the effectiveness of removing contamination, a modified method from Rajala et al. (2013) was used where three symptomatic and three asymptomatic samples were placed in distilled water and vortexed at minimum speed for 10 mins. Four microliters of the rinse solution then served as template for PCR amplification. DNA was amplified using primers ITS1 and ITS4 [26] following methods by [27]. Amplification was observed in symptomatic needles but not in asymptomatic needle samples. Amplification in symptomatic needles was expected since many $L$. concolor and L. montivaga hysterothecia were mature by the time of collection and thus spores were likely easily dispersed in water during vortexing.

\section{Metabarcoding and metatranscriptome sequencing}


DNA and RNA were extracted from asymptomatic and symptomatic needles combined per tree using a combination of methods by [28] and [29] (Supporting 
Table 1

Asymptomatic and symptomatic needle samples from Pinus contorta individual trees that were infected with

Lophodermella needle cast pathogens in various sites in Gunnison National Forest, Colorado, USA. Asterisk (*) indicates the 10 samples that have next generation RNA sequences. Cross $(\dagger)$ and double cross $(\dagger+)$ indicate samples excluded from the metabarcoding or metatranscriptome analysis, respectively.

\begin{tabular}{|c|c|c|c|c|c|c|c|}
\hline \multirow[t]{2}{*}{ Site } & \multirow[t]{2}{*}{ Location } & \multirow[t]{2}{*}{ Tree } & \multicolumn{2}{|c|}{ Lophodermella concolor } & \multirow[t]{2}{*}{ Tree } & \multicolumn{2}{|c|}{ Lophodermella montivaga } \\
\hline & & & Asymptomatic & Symptomatic & & Asymptomatic & Symptomatic \\
\hline \multirow{3}{*}{$\begin{array}{l}\text { Cold Spring } \\
\text { Campground }\end{array}$} & \multirow{2}{*}{$\begin{array}{l}\mathrm{N} \\
38 \circ 46.059^{\prime}\end{array}$} & 1 & $\mathrm{CS} 1-19 \mathrm{CN}^{\dagger}$ & CS01-19CP & & - & - \\
\hline & & 2 & CS02-19CN & CS02-19CP & & - & - \\
\hline & $\begin{array}{l}W \\
106 \circ 38.665^{\prime}\end{array}$ & 3 & CS02-18CN* & CS02-18CP* & & - & - \\
\hline \multirow{3}{*}{$\begin{array}{l}\text { Lakeview } \\
\text { Campground }\end{array}$} & \multirow{3}{*}{$\begin{array}{l}\mathrm{N} \\
38 \circ 49.022^{\prime} \\
W \\
\text { 106॰34.796' }\end{array}$} & 1 & LV01-19CN & LV01-19CP & 1 & LV02-18MN* & LV02-18MP* \\
\hline & & 2 & LV03-19CN & LV03-19CP & 2 & LV03-18MN* & LV03-18MP \\
\hline & & 3 & LV05-19CN & LV05-19CP & & - & - \\
\hline \multirow{5}{*}{$\begin{array}{l}\text { Lodgepole } \\
\text { Campground }\end{array}$} & \multirow{5}{*}{$\begin{array}{l}\mathrm{N} \\
38 \circ 45.733^{\prime} \\
W \\
106 \circ 39.714^{\prime}\end{array}$} & 1 & LP01-19CN & LP01-19CP & & - & - \\
\hline & & 2 & LP02-19CN & LP02-19CP & & - & - \\
\hline & & 3 & LP04-19CN & LP04-19CP & & - & - \\
\hline & & 4 & LP05-19CN & LP05-19CP & & - & - \\
\hline & & 5 & LP06-19CN & LP06-19CP & & - & - \\
\hline \multirow{3}{*}{$\begin{array}{l}\text { Marshall } \\
\text { Pass }\end{array}$} & \multirow{3}{*}{$\begin{array}{l}\mathrm{N} \\
38 \circ 23.289^{\prime} \\
\text { W106॰14.5' }\end{array}$} & 1 & MP01-19CN & MP01-19CP & & - & - \\
\hline & & 2 & MP02-19CN & MP02-19CP & & - & - \\
\hline & & 3 & MP03-19CN & МР03-19СР & & - & - \\
\hline \multirow{6}{*}{$\begin{array}{l}\text { North } \\
\text { Cumberland }\end{array}$} & \multirow{6}{*}{$\begin{array}{l}\mathrm{N} \\
38 \circ 23.289^{\prime} \\
\text { W106॰14.5' }\end{array}$} & 1 & NC02-19CN & NC02-19CP & 1 & $\mathrm{NC} 1-19 \mathrm{MN}^{\dagger}$ & NC01-19MP \\
\hline & & 2 & NC03-19CN & NC03-19CP & 2 & NC04-18MN & $\begin{array}{l}\mathrm{NCO4}^{-} \\
18 \mathrm{MP}^{+\dagger}\end{array}$ \\
\hline & & 3 & NC04-19CN & NC04-19CP & 3 & NC07-19MN & NC07-19MP \\
\hline & & 4 & NC11-19CN & NC11-19CP & 4 & NC11-19MN & NC11-19MP \\
\hline & & 5 & NC13-19CN & NC13-19CP & 5 & NC13-19MN & NC13-19MP \\
\hline & & & - & -- & 6 & NC15-19MN & NC15-19MP \\
\hline \multirow[t]{3}{*}{ Oh Be Joyful } & \multirow{3}{*}{$\begin{array}{l}\mathrm{N} \\
38 \circ 54.840^{\prime} \\
W \\
\text { 107॰01.963' }\end{array}$} & 1 & OBJ01-19CN & OBJ01-19CP & 1 & OBJ09-18MN & OBJ09-18MP \\
\hline & & 2 & OBJ09-18CN & OBJ09-18CP & 2 & OBJ12-19MN & OBJ12-19MP \\
\hline & & 3 & OBJ10-19CN & OBJ10-19CP & & - & - \\
\hline \multirow[t]{4}{*}{ Pitkin } & \multirow{4}{*}{$\begin{array}{l}\mathrm{N} \\
38 \circ 37.850^{\prime} \\
\mathrm{W} \\
106 \circ 28.314^{\prime}\end{array}$} & 1 & PT01-19CN & РТ01-19CP & 1 & PT02-19MN & PT02-19MP \\
\hline & & 2 & PT07-19CN & РТ07-19CP & 2 & PT08-18MN & РT08-18MP \\
\hline & & 3 & PT08-19CN & РТ08-19СР & & - & - \\
\hline & & 4 & PT10-19CN & РТ10-19CP & & - & - \\
\hline
\end{tabular}




\begin{tabular}{|c|c|c|c|c|c|c|c|}
\hline & & 5 & PT11-19CN & \multicolumn{2}{|l|}{ РТ11-19CP } & - & - \\
\hline & & 6 & PT13-19CN & PT13-19CP & & - & - \\
\hline & & 7 & PT15-19CN & PT15-19CP & & - & - \\
\hline \multirow[t]{4}{*}{ Slate River } & \multirow{4}{*}{$\begin{array}{l}N \\
38 \circ 54.186^{\prime} \\
W \\
107 \circ 01.065^{\prime}\end{array}$} & 1 & SR01-19CN & SR01-19CP & 1 & SR09-18MN* & SR09-18MP* \\
\hline & & 2 & SR04-19CN & SR04-19CP & 2 & SR1X-19MN & SR1X-19MP \\
\hline & & 3 & SR05-19CN & SR05-19CP & & - & - \\
\hline & & 4 & SR10-19CN & SR10-19CP* & & - & - \\
\hline \multirow[t]{10}{*}{ Tincup } & \multirow{10}{*}{$\begin{array}{l}\mathrm{N} \\
38 \circ 45.053^{\prime} \\
W \\
106 \circ 28.245^{\prime}\end{array}$} & 1 & TC01-19CN* & ТC01-19CP & 1 & TC01-19MN & TC01-19MP \\
\hline & & 2 & TC02-19CN & TC02-19CP & 2 & TC03-19MN & TC03-19MP \\
\hline & & 3 & TC03-19CN & ТС03-19CP & 3 & TC09-18MN & TC09-18MP \\
\hline & & 4 & TC04-19CN & TC04-19CP & & - & - \\
\hline & & 5 & TC05-19CN & TC05-19CP & & - & - \\
\hline & & 6 & TC07-19CN & ТC07-19CP & & - & - \\
\hline & & 7 & TC08-19CN & ТC08-19CP & & - & - \\
\hline & & 8 & TC09-19CN & ТC09-19CP & & - & - \\
\hline & & 9 & TC10-19CN & TC10-19CP & & - & - \\
\hline & & 10 & TC11-19CN & TC11-19CP & & - & - \\
\hline
\end{tabular}

Information Methods S1). DNA from 60 asymptomatic and 60 symptomatic needle samples were sent to the Genomics Center of the University of Minnesota, Minneapolis, Minnesota, USA for ITS sequencing. Fungal communities were determined by sequencing the ITS2 region [ITS3: (GCATCGATGAAGAACGCAGC) and ITS4: (TCCTCCGCTTATTGATATGC)] and reads were generated using Illumina MiSeq. Selected RNA samples from 5 asymptomatic and 5 symptomatic needle samples were sent to Novogene Corporation, Inc. for library preparation and sequencing (Table 1). Oligo (dT) beads and Ribo-Zero kit were used to enrich eukaryotic mRNA and remove rRNA, respectively. Raw reads from ITS and RNA sequencing were deposited to NCBI SRA database (BioProject ID Number PRJNA753461).

\section{Metabarcoding Analysis}

Quality of ITS reads were assessed using FastQC (v0.11.9) [30]. Samples with $\leq 581$ reads (NC01-19MN and CS01$19 \mathrm{CN}$ ) were excluded from the subsequent analyses. Reads were trimmed using Trimmomatic (v. 0.36) [31] to retain only those with minimum length of $150 \mathrm{bp}$, and a threshold of 15 for end bases and average quality within a 5-base window. Further sequence processing was conducted using Mothur (v.1.40.5) [32] following the MiSeq standard operating procedure (accessed 04/2020) as well as protocols developed by [33]. Contigs with a length $\geq 426 \mathrm{bp}$, and those containing ambiguous bases and homopolymers $\geq 8 \mathrm{bp}$ were discarded. Sequences associated with chloroplast, mitochondria, archaea, and bacteria lineages were removed from the table of classified sequences. UCHIME [34] was used to de novo identify and remove chimeric sequences. USEARCH, utilizing the dgc (distancebased greedy clustering) option, was used for clustering. Groups that were at least $97 \%$ similar were classified to belong to the same operational taxonomic unit (OTU). Sequences were assigned to their taxonomic units using Wang's Naïve Bayes classifier with a cutoff value of 80 [35] and utilizing Mothur UNITE+INSD dataset (v.04022020) 
[36] with additional Lophodermella ITS dataset. The statistical program R (v3.5.0), with the vegan package [37] and software packages metagenomeseq [38] and phyloseq [39], were used to analyze the data from Mothur. OTUs with low number of counts $(\leq 10)$ were first removed to decrease error rate. Sequence depth and rarefaction curves were then obtained, using vegan, to assess whether the depth of sequences was sufficient to provide reasonable evaluation of the fungal diversity within samples. After merging OTUs that were similar at certain taxonomic ranks using the phyloseq package, the distribution of taxa with at least $1 \%$ proportion across treatments was assessed through bar plots.

To analyze the significance of the interaction between treatments on the alpha and beta diversity, only those samples in sites that have both L. concolor and L. montivaga were considered in the succeeding statistical analyses (Table 1). Alpha diversity measures (Shannon and inverse Simpson indices) were generated using the estimate_richness function in phyloseq. Rarified richness was obtained through vegan. Linear models were fitted for richness, and Shannon and inverse Simpson diversity indices. Inverse Simpson diversity index was log-transformed to fit model assumptions. The interaction between pathogen species (L. concolor and L. montivaga) and disease symptoms (asymptomatic and symptomatic) was included as a variable alongside site as covariate. The Im and Anova functions from the car package [40] were used to fit the model. The emmeans function from the emmeans package [41] was used to perform pairwise comparisons.

To analyze differences in beta diversity, Principal Coordinates Analysis (PCoA) was performed using the vegan package where dissimilarity was calculated using Bray-Curtis distance. A constant was added using the [42] method to correct for negative eigenvalues. To determine the differential endophyte community composition using relative abundances of OTUs, permutational multivariate analysis of variance (PERMANOVA) was implemented using adonis2 function also from vegan. Marginal effect of the interaction between pathogen species and disease symptoms was assessed for potential significant impact while site was used to constrain permutations.

To investigate further the taxonomic identities of OTUs assigned as 'Fungi unclassified,' these contigs were BLAST against the NCBI-nt database. As $96 \%$ of 'Fungi unclassified,' had BLAST hits to non-fungal lineages, a separate set of analyses was performed where contigs assigned as 'Fungi unclassified' and non-fungal lineages were removed from the table of classified sequences. Subsequent statistical analyses, including alpha and beta diversity analyses, were conducted using the newly derived data.

\section{Metatranscriptome analysis}

Quality of forward and reverse RNA sequences was evaluated by Novogene and using FastQC [30]. Raw Illumina reads containing adapter contamination, $>10 \%$ uncertain nucleotides, and $>50 \%$ low quality nucleotides (Qscore $\leq 5$ ) among samples were removed by Novogene. No filtered reads across samples were further trimmed due to high quality Phred scores (> 30) during FastQC visual inspection. De novo metatranscriptome assembly was performed using the Trinity software (v2.11.0) [43]. To examine the representation of reads in the assembly, bowtie2 was used to capture and count the reads from individual samples that mapped back to the metatranscriptome assembly. TransRate [44] was also used to examine the quality of the de novo assembly. Transcript abundance was estimated using the RNA-Seq by Expectation Maximization (RSEM v1.3.3) [45]. Correlation analyses of biological replicates between and across treatments were performed using the Pearson correlation matrix to check for outlier samples. Due to the low sum of mapped fragments $(<5 e+6)$ and correlation value $(<0.02)$, NC04-18MP was removed from the subsequent analyses.

Differential expression of transcripts was analyzed using edgeR (v3.32.1) [46]. The count table of the Trinity transcript (isoform) abundance was filtered at counts per million (CPM) of 1 and transcripts must be present in at least 1 
replicate. Significantly differentially expressed (DE) transcripts were determined using the following parameters: 0.05 level of significance after using false discovery rate (FDR) for multiple testing, and a minimum fold change of 2 . Data were normalized using trimmed mean of $\mathrm{M}$ values (TMM) and fitted in a generalized linear model with four contrast arguments: (1) L. concolor asymptomatic (LC_ASYM) vs. L. concolor symptomatic (LC_SYM), (2) L. montivaga asymptomatic (LM_ASYM) vs. L. montivaga symptomatic (LM_SYM), (3) L. concolor asymptomatic (LC_ASYM) vs. L. montivaga asymptomatic (LM_ASYM), and (4) L. concolor symptomatic (LC_SYM) vs. L. montivaga symptomatic (LM_ASYM).

SortMeRNA was used to distinguish the non-coding regions (rRNA) among the DE transcripts [47]. The longest open reading frames of coding regions were predicted using orfipy [48]. The resulting protein products were then subject to MMseqs2 search [49] against the following databases to predict the taxonomy, protein domains, and proteins related to pathogen-host interactions: concatenated databases of NCBI nr [50] and published genome sequences including those of Rhytismataceae species stored at JGI Mycocosm [51], PfamA full 2021 [52], and PHI-base 4.10 [53], respectively. Overall, only the annotated transcripts with an e-value $<1 \mathrm{e}-05$ were considered. Transcripts with coverage and identity $\geq 50 \%$ in the concatenated NCBI nr and JGI Mycocosm database and PHI-base were retained. This concatenated database was used to sort fungal and plant transcripts.

Effectors (with $\geq 95 \%$ probability) and carbohydrate active enzymes (CAZymes) were predicted using EffectorP 2.0 [54] and dbCAN2 [55], respectively. Within dbCAN2, transcripts with hits in at least two of the three databases (HMMER, DIAMOND and Hotpep) were considered. Metabolic pathways of predicted proteins (FDR < 0.05$)$ were searched through BlastKOALA [56] using the family_eukaryotes KEGG database with the 'fungi' or 'plant' taxonomy option [57]. Orthologous gene clusters among treatments and their gene ontology (GO) annotations were determined through Orthovenn2 [58] with an e-value of < 1e-2 and inflation value of 1.5. Expressed plant receptor genes with > 50\% identity were determined through PRGdb 3.0 [59]. DeepLoc 1.0 [60] was used to predict secreted proteins.

\section{Results}

\section{Mycobiome sequences, composition and diversity}

A total of 7,670,091 ITS contigs were obtained from the DNA of 60 symptomatic and 58 asymptomatic needle samples obtained after screening and filtering. Of these, 705,680 were unique contigs which were then further reduced to 664,848 unique contigs with a total of 7,436,294 sequences after removing chimeras and non-fungal lineages. There were only 1,246 OTUs out of 11,691 with $>10$ counts, representing a total of $7,279,077$ contigs across all samples. Of these OTUs, the majority (80\%) fell under Ascomycota while $18 \%$ remained unclassified. These OTUs classified as fungi represented 159 species, 206 genera, 142 families, 62 orders and 20 classes. However, of the 260,014 contigs without no specific fungal classification, only $2 \%$ belonged to unclassified fungal lineage based on BLAST hits against NCBI database while a majority (96\%) belonged to non-fungal lineages and $2 \%$ had no taxonomic assignments.

Of the fungal OTUs classified using UNITE database, Lophodermella concolor and L. montivaga dominated in their respective symptomatic needles by $67 \%$ and $96 \%$, respectively (Fig. 1) followed by unclassified Ascomycota with an average of $5 \%$, Sydowia polyspora (4\%) and unclassified Cladosporium (2\%). While one Lophodermella pathogen dominated, a low proportion $(\leq 1 \%)$ of the other Lophodermella species was observed in symptomatic needles. Unclassified Ascomycota dominated among asymptomatic needles with an average of $18.1 \%$ followed by $S$. polyspora (18\%) and unclassified Cladosporium (5\%). Interestingly, L. concolor (19\%) and L. montivaga (7\%) were both present in asymptomatic needles albeit in low numbers relative to their symptomatic needle counterparts. 
We found that the pathogen species and disease symptoms, accounting for site variations, were significant predictors in both alpha and beta diversity (Supporting Information Table S1). A significantly higher diversity was observed in asymptomatic needles compared to their symptomatic counterparts (Table 2), suggesting colonization of a variety of fungal species. While differences in diversity among asymptomatic needles was marginal (adjusted $p$-value $>0.05$ ), the diversity between needles that were symptomatic of $L$. concolorand $L$. montivaga were profoundly different (adjusted p-value $<0.05$ ). Meanwhile, $29 \%$ of the variability was explained by pathogen and disease symptoms (Fig. 2). Despite removing contigs that matched to 'Fungi unclassified,' diversity remained significantly different between asymptomatic needles vs. symptomatic needles, and between needles symptomatic of L. concolor and L. montivaga (Supporting Information Table S2).

Table 2

Diversity measures among asymptomatic and symptomatic Pinus contorta needles collected from Gunnison National Forest, Colorado, USA

\begin{tabular}{|c|c|c|c|c|}
\hline Needle Treatment & Species & Predicted means (se) & t-ratio & p-value \\
\hline \multicolumn{5}{|l|}{ Richness } \\
\hline \multirow[t]{2}{*}{ Asymptomatic } & L. concolor & $66.78(4.02)$ & \multirow[t]{2}{*}{-0.123} & \multirow[t]{2}{*}{0.9025} \\
\hline & L montivaga & $67.62(5.57)$ & & \\
\hline \multirow[t]{2}{*}{ Symptomatic } & L. concolor & $35.94(4.02)$ & \multirow[t]{2}{*}{3.842} & \multirow[t]{2}{*}{0.0002} \\
\hline & L montivaga & $9.71(5.57)$ & & \\
\hline \multicolumn{5}{|l|}{ Shannon Index } \\
\hline \multirow[t]{2}{*}{ Asymptomatic } & L. concolor & 2.13 (0.09) & \multirow[t]{2}{*}{-0.157} & \multirow[t]{2}{*}{0.8756} \\
\hline & L montivaga & $2.16(0.13)$ & & \\
\hline \multirow[t]{2}{*}{ Symptomatic } & L. concolor & $1.20(0.09)$ & \multirow[t]{2}{*}{5.799} & \multirow[t]{2}{*}{$<0.0001$} \\
\hline & L montivaga & $0.28(0.13)$ & & \\
\hline \multicolumn{5}{|c|}{ Inverse Simpson Index } \\
\hline \multirow[t]{2}{*}{ Asymptomatic } & L. concolor & $0.68(0.04)$ & \multirow[t]{2}{*}{-0.604} & \multirow[t]{2}{*}{0.547} \\
\hline & L. montivaga & $0.72(0.05)$ & & \\
\hline \multirow[t]{2}{*}{ Symptomatic } & L. concolor & $0.32(0.04)$ & \multirow[t]{2}{*}{4.352} & \multirow[t]{2}{*}{$<0.0001$} \\
\hline & L. montivaga & $0.06(0.05)$ & & \\
\hline
\end{tabular}

\section{Metatranscriptome Assembly}

The metatranscriptome libraries from the RNA of asymptomatic and symptomatic needle samples generated a total of 237,903,220 reads (Table 3). The assembly generated 2,079,387 transcript contigs with an average length of 552 bases. Fifty percent of the metatranscriptome sequence was covered by contigs with at least 765 bases (N50). More than $86 \%$ of the reads across all samples aligned back to the assembly (Table 3). Correlation assessment between samples within treatments and Principal Component Analysis (Supporting Information Fig. S1) identified NC04-18MP sample as an outlier and thus was excluded from further analysis. The average Pearson correlation between samples within a treatment were 0.8 for LM_SYM (excluding NC04-18MP), 0.6 for LM_ASYM, 0.7 for LC_SYM and 0.7 for 
LC_ASYM. Notably, LC_ASYM and LM_ASYM samples were highly correlated compared to their symptomatic counterpart. The 5 asymptomatic and 4 symptomatic samples, which contained a total of 510,575 fungal OTUs, had 10,505,207 transcripts (Supporting Information Table S3).

\section{Metatranscriptome differential gene expression}

Differential expression profiles were similar across LC_ASYM and LM_ASYM samples (Fig. 3). In contrast, differential expression profiles between LC_SYM and LM_SYM were distinct from each other. A total of 85,798 transcripts were differentially expressed (DE) across all four comparisons: LC_ASYM_vs._LC_SYM, LM_ASYM_vs._LM_SYM, LC_ASYM_vs._LM_ASYM, and LC_SYM_Vs._LM_ASYM. Collapsing identical transcripts produced a total of 51,363 transcripts with $93 \%(47,812)$ classified as non-rRNA.

Table 3

Metabarcoding and metatranscriptome profile of Pinus contorta needle samples. Cross $(\dagger)$ represents samples excluded from the analyses. Proportions (\%) of raw reads that aligned to the metatranscriptome assembly and the overall alignment rate are also shown.

\begin{tabular}{|c|c|c|c|c|c|c|}
\hline \multirow[t]{2}{*}{$\begin{array}{l}\text { Disease } \\
\text { symptom }\end{array}$} & \multirow[t]{2}{*}{ Sample } & \multicolumn{2}{|c|}{$\begin{array}{l}\text { DNA contigs } \\
\text { (metabarcoding) }\end{array}$} & \multirow[t]{2}{*}{$\begin{array}{l}\text { Raw RNA } \\
\text { reads* }\end{array}$} & \multirow{2}{*}{$\begin{array}{l}\text { Paired reads that } \\
\text { aligned concordantly to } \\
\text { the assembly } \geq 1 \text { time } \\
(\%)\end{array}$} & \multirow{2}{*}{$\begin{array}{l}\text { Overall rate (\%) } \\
\text { of alignment to } \\
\text { the assembly }\end{array}$} \\
\hline & & $\begin{array}{l}\text { L. } \\
\text { concolor }\end{array}$ & $\begin{array}{l}\text { L. } \\
\text { montivaga }\end{array}$ & & & \\
\hline \multirow[t]{2}{*}{ Asymptomatic } & $\begin{array}{l}\text { CSO2- } \\
18 C N\end{array}$ & 1,014 & 173 & $21,337,962$ & 78.82 & 93.4 \\
\hline & $\begin{array}{l}\text { TC01- } \\
19 C N\end{array}$ & 2,386 & 3,706 & $24,537,902$ & 79.75 & 93.56 \\
\hline \multirow[t]{2}{*}{ Symptomatic } & $\begin{array}{l}\text { CSO2- } \\
18 C P\end{array}$ & 26,149 & 84 & $20,416,050$ & 66.61 & 87.89 \\
\hline & $\begin{array}{l}\text { SR10- } \\
\text { 19CP }\end{array}$ & 22,791 & 0 & $21,485,100$ & 71.65 & 91.68 \\
\hline \multirow[t]{3}{*}{ Asymptomatic } & $\begin{array}{l}\text { LVO2- } \\
18 \mathrm{MN}\end{array}$ & 22,078 & 18,744 & $22,184,468$ & 78.33 & 89.96 \\
\hline & $\begin{array}{l}\text { LVO3- } \\
18 \mathrm{MN}\end{array}$ & 522 & 4,785 & $25,400,132$ & 73.79 & 86.48 \\
\hline & $\begin{array}{l}\text { SR09- } \\
\text { 18MN }\end{array}$ & 26,245 & 1,422 & $30,543,904$ & 77.44 & 94.48 \\
\hline \multirow[t]{3}{*}{ Symptomatic } & $\begin{array}{l}\text { LV02- } \\
\text { 18MP }\end{array}$ & 0 & 20,353 & $20,609,968$ & 79.88 & 92.06 \\
\hline & $\begin{array}{l}\text { NC04- } \\
18 \mathrm{MP}^{\dagger}\end{array}$ & 3 & 63,789 & $24,456,808$ & 77.26 & 92.70 \\
\hline & $\begin{array}{l}\text { SR09- } \\
\text { 18MP }\end{array}$ & 0 & 43,121 & $26,930,926$ & 78.37 & 92.76 \\
\hline
\end{tabular}

The largest number of DE transcripts was observed in LC_SYM_vs._LM_SYM $(38,439)$, followed by LM_ASYM_vs._LM_SYM (32,779) and LC_ASYM_vs._LC_SYM $(14,562)$. Only 18 DE transcripts were found in the 
LC_ASYM_vs._LM_ASYM comparison. Greater expression of transcripts was observed in symptomatic needles compared to asymptomatic ones: 14,433 (99\%) in LC_SYM vs. 129 (1\%) in LC_ASYM, and 32462 (99\%) in LM_SYM vs. 317 (1\%) in LM_ASYM. Notably, of the 46,895 DE transcripts in needles symptomatic of L. concolor and L.montivaga, only $1.1 \%$ were shared between species. Similarly, needles asymptomatic of $L$. concolor and $L$. montivaga shared only $0.9 \%$ of the $446 \mathrm{DE}$ transcripts. These may be attributed to possible sequence divergence that separated the orthologs into distinct transcripts. Orthovenn analysis showed that symptomatic needles contained more transcripts that belong to unique protein clusters than in asymptomatic needles, with only $1 \%$ shared between them (Supporting Information Fig. S2). Nearly 98\% of the total protein clusters in LC_ASYM_vs._LC_SYM $(2,680)$ and LM_ASYM_Vs._LM_SYM $(5,679)$ were exclusive to symptomatic needles.

Of the 85,798 DE transcripts, only 39,807 had taxonomic annotations with $61 \%$ identified as fungi. Nearly $30 \%$ and $3 \%$ of these DE transcripts were identified as bacteria and plants, respectively (Supporting Information Fig. S3). The majority (98\%) of the 24,289 fungal transcripts belonged to Ascomycota, followed by Basidiomycota (1.1\%) and Mucoromycota (0.4\%). None of the DE transcripts in LC_ASYM_vs._LM_ASYM were identified as fungi. Among the comparisons involving asymptomatic vs. symptomatic needles, no fungal taxa with > $10 \mathrm{DE}$ transcripts were found in asymptomatic needles (Supporting Information Table S4). However, fungal transcripts may still be present but were not significantly expressed. In symptomatic needles, many fungal DE transcripts (47\% and 49\% in LC_SYM and LM_SYM, respectively) were classified under Rhytismataceae (Fig. 4). Since there are no available sequenced

\section{Lophodermella genomes, rhytismataceous transcripts could only be matched to other closely related genera (Supporting Information Table S4).}

Differentially expressed plant transcripts were more abundant in symptomatic needles than their asymptomatic counterparts (Supporting Information Fig. S3), with nearly twice the DE transcript count to that of asymptomatic needles. Out of the total 1,317 DE plant transcripts across all four comparisons, only $31 \%$ were classified as conifers. These conifer transcripts, dominated by Picea sitchensis, were more abundant in asymptomatic needles within comparisons LC_ASYM_Vs._LC_SYM and LM_ASYM_Vs._LM_SYM. In contrast, transcripts classified as non-conifers were generally abundant in symptomatic needles possibly due to the lack of genome annotation for $P$. contorta host.

\section{Functional annotation of fungal transcripts}

To obtain insights into the metabolic activities of the mycobiome within healthy and diseased needles, DE fungal transcripts were compared in functional annotation databases: Pfam, PHI-base, EffectorP, dbCAN, KEGG, and SwissProt and GO through Orthovenn. Roughly $80 \%$ of the total DE fungal transcripts across all three comparisons (LC_ASYM_Vs.LC_SYM, LM_ASYM_Vs._LM_SYM and LC_SYM_VS._LM_SYM) had annotations in at least one of the databases, and many of these were highly expressed in symptomatic needles (Figs. 5-7). There were no DE transcripts identified as fungi in LC_ASYM_vs._LM_ASYM. In LC_SYM_vs._LM_SYM comparison, functions of the DE transcripts in both treatments were similar (Figs. 5-7) despite a low proportion of shared transcripts possibly influenced by sequence divergence between the two dominant Lophodermella pathogens.

The fungal community in symptomatic needles highly expressed carbohydrate-active and protein-degrading enzymes (Fig. 5; Supporting Information Table S5). Fungal proteases and peptidases commonly found in symptomatic needles included metallopeptidases, cysteine peptidases, and serine carboxypeptidase (Supporting Information Table S5). While there was remarkable expression of enzymes involved in carbohydrate synthesis, other CAzymes with known functions of lignin and carbohydrate degradation were also common in symptomatic needles. GHs were overrepresented in symptomatic needles (LC_SYM and LM_SYM). Among comparisons of LC_ASYM_Vs._LC_SYM and 
LM_ASYM_vs._LM_SYM, a higher expression of CAzymes, proteases and peptidases was observed in LM_SYM than in LC_SYM. Similarly, lignin degrading enzymes (i.e., AAs 1, 2, 3 and 5) were more abundant in LM_SYM.

Genes related to pathogenicity were also expressed in symptomatic needles (Fig. 6). In asymptomatic vs. symptomatic needle comparisons, more than $50 \%$ of the 604 and 1,235 PHI-base hits in L. concolor (64\%) and L. montivaga (59\%) symptomatic needles, respectively, were associated with pathogenicity, virulence, and/or chemical resistance. Of these, only 6 and 26 L. concolor and L. montivaga transcripts, respectively, were predicted as secreted proteins. Moreover, only 1 out of 5 and 1 out of 3 EffectorP-annotated DE transcripts were predicted as secreted effectors in L. concolor and L. montivaga symptomatic needles, respectively, using DeepLoc. In contrast, no significant DE transcripts in asymptomatic needles had hits to EffectorP nor PHI-base databases, suggesting no significant pathogenic activity in asymptomatic needles.

KEGG annotations of fungal transcripts also showed enhanced gene expression in symptomatic needles compared to asymptomatic ones. Genes involved in the synthesis of secondary metabolites were also highly expressed in symptomatic needles (Fig. 7). Apart from terpenoids backbone synthesis (KO00900), other dominant proteins in symptomatic needles were involved in the synthesis of other secondary metabolites such as phenylpropanoid (KO00942), isoquinoline alkaloid (KO00950), tropane, piperidine and pyridine alkaloid (KO00960), and streptomycin (KO00521) (Supporting Information Table S6). Interestingly, pathways in symptomatic needles also included membrane transport (KO02010 ABC transporters: LC_SYM=14, LM_SYM=31) and environmental adaptation (KO04626 Plant-pathogen interaction: LC_SYM=2, LM_SYM=1). A high expression of genes involved in xenobiotics biodegradation and metabolism were also observed in symptomatic needles.

More GO annotations were detected in symptomatic needles that were related to biological processes, molecular functions and cellular components observed (Fig. 8). The only two fungal DE transcripts in LM_ASYM in comparison LM_ASYM_Vs._LM_SYM were related to biological processes (i.e., xanthophyll cycle and transcription elongation). Interestingly, of the biological processes identified in symptomatic needles, processes such as protein and membrane transport, ubiquitin-dependent protein catabolic process, and pathogenesis were among the most common. Further, molecular functions common in symptomatic needles include oxidoreductase and metallopeptidase activities, and zinc and metal ion binding. Gene products or protein complexes in symptomatic needles were commonly observed in the cellular membrane.

\section{Functional annotation of plant transcripts}

For DE transcripts taxonomically annotated as plants, functional annotation was conducted using the following protein databases: Pfam, dbCAN, KEGG, PRGdb, NCBI, and Swiss-Prot and GO in Orthovenn. Seventy-eight percent of the total 1,317 plant DE transcripts across all four comparisons had annotations in at least one of the functional annotation databases, although a few (25 DE transcripts) were annotated as proteins with uncharacterized, unknown or hypothetical function.

DE plant transcripts in both asymptomatic and symptomatic needles mostly belonged to glycosyl hydrolase and glycosyltransferase families. However, only a small proportion of transcripts (3\% in LC_ASYM vs. LC_SYM and 6\% in LM_ASYM vs. LM_SYM) were annotated using dbCAN2. Interestingly, albeit in low numbers, protein-degrading enzymes such as metallopeptidases (peptidase family M20/M25/M40, M24 and M41) and serine peptidases (peptidase S26, serine aminopeptidase S33, X-Pro dipeptidyl-peptidase S15) were found in asymptomatic needles but not in symptomatic ones. 
PRGdb annotation further showed higher expression of plant resistance genes in asymptomatic needles compared to symptomatic ones (Supporting Information Table S7). Kinase (KIN) with transmembrane (TM) and kinase domains was the most dominant class in asymptomatic needles followed by receptor-like proteins (RLP) with TM and extracellular leucine-rich repeat (LRR) domains. Similarly, annotated through KEGG database, abundant proteins associated with the mitogen-activated protein kinase (MAPK) signaling pathway and plant hormone signal transduction were more abundant in asymptomatic needles. Phospholipase D signaling pathway was unique to asymptomatic needles.

In contrast, the DE transcripts associated with other pathways for signal transduction were slightly more abundant in symptomatic needles (Supporting Information Table S8). About 37\% of the DE transcripts in needles symptomatic of L. concolor (47 out of 127) and L. monitvaga (98 out of 265) were annotated through KEGG. However, of these, only 5 were common, which included ATP citrate lyase (ACL; K01648) and translation initiation factor 5A (elF5A; K03262) that were exclusive to symptomatic needles, and serine/threonine-protein phosphatase 2A (PP2A; K04354) and cleavage and polyadenylation specificity factor (CPSF; K14404) which were abundant in symptomatic needles. DE plant proteins in both asymptomatic and symptomatic needles were mostly related to biological processes, although GO annotated proteins were generally more abundant in symptomatic needles (Fig. 9). Interestingly, in LC_SYM_vs._LM_SYM, plant proteins highly expressed in LC_SYM were related to response to stresses such as salt stress (11) and oxidative stress (4) whereas LM_SYM was mostly dominated by proteins related to pathogenesis (12) and transmembrane transport (9).

\section{Functional annotation of other transcripts}

Despite the enrichment of eukaryotic organisms, bacterial transcripts were still recovered with a majority observed in symptomatic needles (LC_ASYM=2 and LC_SYM=2,0687 in LC_ASYM_vs._LC_SYM, and LM_ASYM=0 and LM_SYM=4,420 in LM_ASYM_vs._LM_SYM). Symptomatic needles contained highly abundant proteins (Supporting Information Table S9) and enzymes for carbohydrate synthesis and degradation (Supporting Information Fig. S4). Interestingly, chitin-degrading enzymes (CE9 and GH125) were enriched, though not the most abundant, among the bacterial transcripts in symptomatic needles. GO annotations also revealed high expression of genes involved in biological processes, particularly protein transport (G0:0015031) and pathogenesis (G0:0009405).

A large proportion (54\%) of the metatranscriptome remained taxonomically unclassified and the majority was identified in symptomatic needles (Supporting Information Fig. S3). Of these, only $8 \%(3,676$ out of 45,991 ) were functionally annotated using Pfam, PHI-base, EffectorP, dbCAN, and Swiss-Prot and GO through Orthovenn. Proteins associated with biological processes were abundant in symptomatic needles within comparisons LC_ASYM_Vs._LC_SYM and LM_ASYM_Vs._LM_SYM. In particular, ubiquitin-dependent protein catabolic process and protein transport were commonly abundant among symptomatic needles. LC_SYM was further dominated with proteins involved in pathogenesis and transcription while transposition and DNA integration were abundant in LM_SYM. Similarly, while only a few, CAzymes that degrade cellulose (AA9) and hemicellulose (GH16) were found in symptomatic needles while only GT1 for carbohydrate synthesis was observed in asymptomatic needles. Interestingly, extracellular effectors were predicted only among transcripts in symptomatic needles (LC_SYM=5, LM_SYM=7).

\section{Discussion}

This study explored mycobiota shifts and changes in gene expression in the interaction among fungal endophytes, Lophodermella pathogens, and $P$. contorta host in the Lophodermella needle cast pathosystem. The results supported our hypothesis and revealed an adverse impact of disease on the needle mycobiota, with a significant decrease of 
fungal diversity as the pathogenic mycobiota dominated by Lophodermella pathogens becomes highly active. It further showed an elicitation of diverse plant defense mechanisms that differed in healthy and diseased needles, and between $L$. concolor and $L$. montivaga dominated mycobiota. This study also described for the first time the endophytic lifestyle of $L$. concolor and L. montivaga.

\section{Mycobiome composition and diversity}

This study showed that $P$. contorta needles host a diverse community of fungal species dominated by Ascomycetes, a group commonly abundant in conifer needle tissues [e.g., 61,62]. Similar to other studies, a large number of fungal OTUs remain unclassified, which highlights the wide diversity of potentially novel endophytes with ecological roles yet to be identified $[2,63]$. This study further identified that $L$. concolor and $L$. montivaga may be part of the 'normal' mycobiome of $P$. contorta needles as both are present in asymptomatic and symptomatic needles. The presence of pathogen in the absence of disease can occur in many pathosystems where potential pathogens are members of a healthy microbiome [64]. Since this study only examined in P. contorta stands in Colorado, whether one or both Lophodermella pathogens are present in healthy needles of other $P$. contorta provenances, which have varying levels of needle cast resistance [65], is still unknown. Alternatively, their existence in asymptomatic needles may not necessarily be part of the commensal microflora but instead are persistent infections due to their ability to evade the host immune response as endophytes [66]. Susceptible hosts that are persistently infected then act as pathogen reservoirs.

Dysbiosis in the mycobiota, which is often the result of environmental disturbances [64,67], is characterized as an overgrowth of pathogens, significant depletion of other taxa, loss of beneficial microbes or a combination of these [68]. This is in contrast to a dense and diverse healthy microbiota under normal colonizing conditions [69]. We found a highly diverse fungal community with a large number of differentially abundant OTUs in asymptomatic needles, which indicates a condition that allows many, and mostly undescribed, fungal species to colonize the needle tissue. However, at the symptomatic state, diversity was significantly reduced in the needle mycobiota with significant enrichment of Lophodermella pathogens. This dominance may be a manifestation of Lophodermella pathogens outcompeting other commensal species in the mycobiota for niche and/or other resources through various strategies $[70,71]$, or a result of environmental changes favoring the growth of pathogens over other endophytes [72].

\section{Fungal gene expressions in the Lophodermella needle cast pathosystem}

Plant pathogens upregulate an arsenal of pathogenicity-related genes that lead to disease emergence and increased severity until host mortality $[73,74]$. This study showed a variety of pathogenicity-related genes (e.g., CAZymes, effectors, secondary metabolites and $A B C$ transporters) that were highly expressed by the mycobiota, mostly Rhytismataceae, at the necrotrophic phase of the disease. These could largely be driven by Lophodermella pathogens as they dominate the mycobiota and colonize the needle tissue. In contrast, little to none of these genes were significantly expressed in asymptomatic needles, which could be an indication of low or absent pathogen activity. This could be part of the cryptic strategy of fungal endophytes after their initial host penetration to evade plant defense responses. Until needle senescence, latent needle pathogens exhibited either no additional growth or a slow continuous growth in intercellular spaces after initial infection $[75,76]$. Thus, the absence of disease in $P$. contorta could be a manifestation of Lophodermella pathogens evading host plant response through their marginal growth.

We found many plant cell wall degrading enzymes expressed in symptomatic needles which likely induced host necrosis and further facilitated pathogen growth. This is similar to previous observations among hemibiotrophic foliar 
pathogens, where cell wall degradation via glycosyl hydrolases were significantly upregulated at the latter stage of disease $[77,78]$. We also found effectors in symptomatic needles, which are likely necrotrophic effectors produced by hemibiotrophic or latent pathogens as they switch to the necrotrophic stage, and thus inducing host cell death [79]. This possibly allowed Lophodermella pathogens access to more nutrients leading to sporulation on needles [80]. Metabolic pathways were also highly active in symptomatic needles, which could indicate abundance of toxins and other metabolites at the pathogenic necrotrophic state that potentially trigger plant hypersensitive response necessary for host invasion and produce reactive oxygen species that inhibit growth of biotrophs [81]. Transport and/or secretion of these metabolites may be facilitated by $A B C$ transporters that were abundant in symptomatic needles, although ABC transporters may also be involved in host penetration, survival and virulence [82-84].

\section{Plant interactions in the Lophodermella needle cast pathosystem}

Plant hosts have a wide ecological spectrum of interactions with the endophytic mycobiota. We observed an overexpression of host defense related genes, including KINs and RLPs, in asymptomatic needles suggesting the recognition of microbial infection and thereby activating the first layer of plant inducible defense $[85,86]$ although RLPs could be involved in cell growth and development [87] as part of the normal functioning of the plant host. Signaling pathways common in asymptomatic needles (e.g., MAPK and phospholipase D) could also be linked to plant hormone signaling and/or transduction with critical roles in plant growth and defense [88, 89].

Albeit rare, protein-degrading enzymes were unique in asymptomatic needles compared to symptomatic needles which could indicate plant's response to improve tolerance against stressors. Some (e.g., prolyl aminopeptidases) can be rapidly over-induced when plants are subject to unfavorable environment $[90,91]$ and improve plant stress tolerance [92]. We also found enzymes (e.g., M41) that could function as response to light stress resulting in photosystem repair [93].

Plants are highly defensive and metabolically active as a response to the necrotrophic growth of hemibiotrophic pathogens [77]. We found that symptomatic needles overly expressed a set of genes related to environmental stress, which may be triggered by the pathogenic activity of the mycobiota. Plants utilize glycoside hydrolases to degrade cell walls in response to stressors such as pathogen invasion and thereby inhibiting further fungal pathogen growth $[94,95]$. The presence of these plant enzymes in symptomatic needles, although rare, could be part of host defense against further growth of Lophodermella pathogens. Similarly, enriched proteins in symptomatic needles that were involved in programmed cell death (CPFS [96], elF5A [97], ACL [98]) and stress signaling (PP2A) [99] are likely an attempt by $P$. contorta to defend itself against the pathogenic activity of the mycobiota. However, this plant defensive environment may only have eventually increased susceptibility and plant necrosis [100], and fungal pathogen growth and development [101].

Despite highly similar functions of genes expressed by L. concolor and L. montivaga at the symptomatic phase, gene expression by the plant differed between L. concolor and L. montivaga symptomatic needles. This could indicate a set of unique and possibly uncharacterized strategies by each Lophodermella pathogen that elicit different responses in $P$. contorta, which could influence important features (e.g., morphology and host specificity) unique to each species. Similar observations were noted in two Phytophthora pathogens of Theobroma cacao where the differences in genome structure and in planta transcriptome expression profiles presumably resulted in differences in host range [102].

\section{Characterization of other transcripts}

Metatranscriptome analyses in other pathosystems have shown a consortium of active microbes that drive disease development $[16,103]$ and suppression [15]. The high expression of non-fungal genes mostly associated with protein 
and substrate breakdown in symptomatic needles could indicate a more complex interaction between disease players (i.e., endophytes, pathogen and host), and thus further investigation needs to be conducted. Interestingly, we found an overexpression of chitin-degrading enzymes at the symptomatic phase which could have likely reduced pathogen activity. Future studies should explore the potential of these beneficial bacterial communities as a strategy to control conifer foliage diseases.

We found an abundance of transcripts with unknown identities and functions, showing the existing limitations and challenges in our understanding about microbial community interactions [104, 105]. Nonetheless, there is little doubt about the role CAZymes and effectors play in the necrosis observed in symptomatic needles. However, whether these were proteins employed by Lophodermella during pathogenesis or by another organism in the community remains unknown. Using sequenced genomes of $L$. concolor and L. montivaga as references in transcriptome assembly would allow us to refine which transcripts belong to Lophodermella species within infected needle community. However, their inability to grow in culture media and lack of asexual reproductive structures present a challenge in genome sequencing. Therefore, either metagenomic or single cell approaches may be necessary to effectively capture and assemble their genomes [106].

\section{Lophodermella as latent pathogens in $\mathrm{P}$. contorta needles}

Several needle pathogens were identified as latent pathogens through isolation from healthy needles of their conifer hosts [1, 107]. In contrast, until recently, there has been no documentation to suggest that endophytic species within the Lophodermella genus exist, possibly owing to their fastidious or likely obligate lifestyle. Previous observations pointed to Lophodermella species being active parasites $[20,23]$ and survival throughout the next season is possible if infected needles are not shed [108]. This present study provided evidence that $L$. concolor and L. montivaga are likely latent needle pathogens in $P$. contorta. Despite their significant presence, we did not detect pathogenic activities in asymptomatic needles which suggests a period of dormancy for Lophodermella pathogens.

With the presence of Lophodermella spp. in healthy and diseased P. contorta needles, what triggers the lifestyle transition of these pathogens and symptom development remains a question. Nonetheless, it has been shown that environmental factors could favor further growth and/or activity of latent pathogens. The enhanced sporulation and infection of Lophodermella pathogens with warm moisture [21] could exacerbate pathogen invasion resulting in an increase in relative abundance. This pathogen excess in host tissue then leads to an intensified disease incidence or severity $[64,109]$ and demonstrates an imbalance in an otherwise balanced system of antagonism between disease players that leads to disease development [110]. [6] further postulated that a rapid change in endophytic density due to adverse biotic and abiotic stresses, results in premature needle cast. Alternatively, latency could be affected by processes involving physiological constraints and/or adaptation [111] or a necessity to switch to necrotrophy for pathogen survival as $\mathrm{H}_{2} \mathrm{O}_{2}$ in plants accumulates [101]. Thus, more work needs to be done to understand the transitional cues involved in this pathosystem that would also incorporate environmental and anatomical factors at different life stages of the pathogens.

\section{Declarations}

\section{Acknowledgements}

The authors would like to thank Kelly S. Burns, Suzanne B. Marchetti and Dr. James J. Worrall of the USDA Forest Service Forest Health Protection - Rocky Mountain Region for their valuable inputs in funding this research and in

Page $16 / 29$ 
field sampling. This project is funded through the USDA Forest Service Forest Health Protection and Special Technology Development Program (STDP-R2-2017-3). The authors are also grateful to the 1000 Fungal Genomes project, especially to the following principal investigators, for access to unpublished genome data: Dr. Richard Hamelin (Elytroderma deformans), Drs. Francis Martin and Marie Leys (Lophodermium piceae), Dr. Ryoko Oono (Lophodermium nitens), and Dr. Joseph Spatafora (Spathularia flavida, Pseudgraphis elatina and Coccomyces strobi). The genome sequence data produced by the Joint Genome Institute, a Department of Energy (DOE) Office of Science User Facility, was supported by the Office of Science of the US DOE under Contract no. DE-AC02-05CH11231.

\section{References}

1. Stone, J. K., Polishook, J. D. \& White, J. F. ENDOPHYTIC FUNGI. in Biodiversity of Fungi 241-270 (Elsevier, 2004). doi:10.1016/B978-012509551-8/50015-5.

2. Rodriguez, R. J., White Jr, J. F., Arnold, A. E. \& Redman, R. S. Fungal endophytes: diversity and functional roles: Tansley review. New Phytologist 182, 314-330 (2009).

3. Terhonen, E., Blumenstein, K., Kovalchuk, A. \& Asiegbu, F. O. Forest Tree Microbiomes and Associated Fungal Endophytes: Functional Roles and Impact on Forest Health. Forests 10, 42 (2019).

4. Gao, F., Dai, C. \& Liu, X. Mechanisms of fungal endophytes in plant protection against pathogens. African Journal of Microbiology Research 4, 1346-1351 (2010).

5. Jia, Q., Qu, J., Mu, H., Sun, H. \& Wu, C. Foliar endophytic fungi: diversity in species and functions in forest ecosystems. Symbiosis 80, 103-132 (2020).

6. Sieber, T. N. Endophytic fungi in forest trees: are they mutualists? Fungal Biology Reviews 21, 75-89 (2007).

7. Slippers, B. \& Wingfield, M. J. Botryosphaeriaceae as endophytes and latent pathogens of woody plants: diversity, ecology and impact. Fungal Biology Reviews 21, 90-106 (2007).

8. Busby, P. E., Peay, K. G. \& Newcombe, G. Common foliar fungi of Populus trichocarpa modify Melampsora rust disease severity. New Phytol 209, 1681-1692 (2016).

9. Ridout, M. \& Newcombe, G. Sydowia polyspora is both a Foliar Endophyte and a Preemergent Seed Pathogen in Pinus ponderosa. Plant Disease 102, 640-644 (2018).

10. Brader, G. et al. Ecology and Genomic Insights into Plant-Pathogenic and Plant-Nonpathogenic Endophytes. Annu. Rev. Phytopathol. 55, 61-83 (2017).

11. Peters, S., Dammeyer, B. \& Schulz, B. Endophyte-host Interactions. I. Plant defense reactions to endophytic and pathogenic fungi. Symbiosis 25, 193-211 (1998).

12. Krings, M. et al. Fungal endophytes in a 400-million-yr-old land plant: infection pathways, spatial distribution, and host responses. New Phytologist 174, 648-657 (2007).

13. Koskella, B., Meaden, S., Crowther, W. J., Leimu, R. \& Metcalf, C. J. E. A signature of tree health? Shifts in the microbiome and the ecological drivers of horse chestnut bleeding canker disease. New Phyto/ 215, 737-746 (2017).

14. Kovalchuk, A. et al. Mycobiome analysis of asymptomatic and symptomatic Norway spruce trees naturally infected by the conifer pathogens Heterobasidion spp.: Mycobiome of Norway spruce. Environmental Microbiology Reports 10, 532-541 (2018).

15. Hayden, H. L., Savin, K. W., Wadeson, J., Gupta, V. V. S. R. \& Mele, P. M. Comparative Metatranscriptomics of Wheat Rhizosphere Microbiomes in Disease Suppressive and Non-suppressive Soils for Rhizoctonia solani AG8. Front. Microbiol. 9, 859 (2018). 
16. Martí, J. M. et al. Metatranscriptomic dynamics after Verticillium dahliae infection and root damage in Olea europaea. BMC Plant Biol 20, 79 (2020).

17. Feau, N. \& Hamelin, R. C. Say hello to my little friends: how microbiota can modulate tree health. New Phyto/ 215, 508-510 (2017).

18. Stewart, J. E., Kim, M.-S., Lalande, B. \& Klopfenstein, N. B. Pathobiome and microbial communities associated with forest tree root diseases. in Forest Microbiology 277-292 (Elsevier, 2021). doi:10.1016/B978-0-12-8225424.00004-8.

19. Carrión, V. J. et al. Pathogen-induced activation of disease-suppressive functions in the endophytic root microbiome. Science 366, 606-612 (2019).

20. Darker, G. The Hypodermataceae of Conifers. vol. 1 (Contributions from the Arnold Arboretum of Harvard University, 1932).

21. Worrall, J. J., Marchetti, S. B. \& Mask, R. A. An Epidemic of Needle Cast on Lodgepole Pine in Colorado. in Biological Evaluation R2-12-01 16 (USDA Forest Service, Rocky Mountain Region, Forest Health Protection, 2012).

22. Abdullah, A. S. et al. Host-Multi-Pathogen Warfare: Pathogen Interactions in Co-infected Plants. Frontiers in Plant Science 8, 1806 (2017).

23. Millar, C. S. Lophodermella species on pines. in In: Recent Research on Conifer Needle Diseases. Eds. Glenn W. Peterson. USDA Forest Service, General Technical Report GTR-WO 50. 45-55 (USDA Forest Service, 1984).

24. Prihatini, I., Glen, M., Wardlaw, T. J. \& Mohammed, C. L. Lophodermium pinastri and an unknown species of Teratosphaeriaceae are associated with needle cast in a Pinus radiata selection trial. For. Path. 45, 281-289 (2015).

25. Rajala, T. et al. Endophyte communities vary in the needles of Norway spruce clones. Fungal Biology 117, 182190 (2013).

26. White, T. J., Bruns, T., Lee, S. \& Taylor, J. Amplification and direct sequencing of fungal ribosomal RNA genes for phylogenetics. in PCR Protocols: A Guide to Methods and Applications. Edited by: Innis MA, Gelfand DH, Sninsky JJ, White TJ 315-322 (Academic Press Inc., 1990).

27. Ata, J. P. et al. Molecular characterization and phylogenetic analyses of Lophodermella needle pathogens (Rhytismataceae) on Pinus species in the USA and Europe. PeerJ 9, e11435 (2021).

28. Zeng, Z., Raffaello, T., Liu, M.-X. \& Asiegbu, F. O. Co-extraction of genomic DNA \& total RNA from recalcitrant woody tissues for next-generation sequencing studies. Future Science OA 4, FSO309 (2018).

29. Cubero, O. F., Crespo, A., Fatehi, J. \& Bridge, P. D. DNA extraction and PCR amplification method suitable for fresh, herbarium-stored, lichenized, and other fungi. Pl Syst Evol 216, 243-249 (1999).

30. Andrews, S. FastQC: a quality control tool for high throughput sequence data. Available online at. http://www.bioinformatics.babraham.ac.uk/projects/fastqc (2010).

31. Bolger, A. M., Lohse, M. \& Usadel, B. Trimmomatic: a flexible trimmer for Illumina sequence data. Bioinformatics 30, 2114-2120 (2014).

32. Schloss, P. D. et al. Introducing mothur: Open-Source, Platform-Independent, Community-Supported Software for Describing and Comparing Microbial Communities. AEM 75, 7537-7541 (2009).

33. Kozich, J. J., Westcott, S. L., Baxter, N. T., Highlander, S. K. \& Schloss, P. D. Development of a Dual-Index Sequencing Strategy and Curation Pipeline for Analyzing Amplicon Sequence Data on the MiSeq Illumina Sequencing Platform. Appl. Environ. Microbiol. 79, 5112-5120 (2013).

34. Edgar, R. C., Haas, B. J., Clemente, J. C., Quince, C. \& Knight, R. UCHIME improves sensitivity and speed of chimera detection. Bioinformatics 27, 2194-2200 (2011). 
35. Wang, Q., Garrity, G. M., Tiedje, J. M. \& Cole, J. R. Naïve Bayesian Classifier for Rapid Assignment of rRNA Sequences into the New Bacterial Taxonomy. AEM 73, 5261-5267 (2007).

36. Abarenkov, K. et al. Full mothur UNITE+INSD dataset 1. (2020) doi:10.15156/BIO/786378.

37. Oksanen, J. et al. Package 'vegan': community ecology package. (2020).

38. Paulson, J. N. et al. metagenomeSeq: Statistical analysis for sparse high-throughput sequencing. (2013).

39. McMurdie, P. J. \& Holmes, S. phyloseq: An R Package for Reproducible Interactive Analysis and Graphics of Microbiome Census Data. PLoS ONE 8, e61217 (2013).

40. Fox, J. \& Weisberg, S. An R Companion to Applied Regression. (2019).

41. Lenth, R., Singmann, H., Love, J., Buerkner, P. \& Herve, M. Package 'emmeans': Estimated Marginal Means, aka Least-Squares Means. (2020).

42. Legendre, P. \& Anderson, M. J. DISTANCE-BASED REDUNDANCY ANALYSIS: TESTING MULTISPECIES RESPONSES IN MULTIFACTORIAL ECOLOGICAL EXPERIMENTS. Ecological Monographs 69, 1-24 (1999).

43. Grabherr, M. G. et al. Full-length transcriptome assembly from RNA-Seq data without a reference genome. Nat Biotechnol 29, 644-652 (2011).

44. Smith-Unna, R., Boursnell, C., Patro, R., Hibberd, J. M. \& Kelly, S. TransRate: reference-free quality assessment of de novo transcriptome assemblies. Genome Res. 26, 1134-1144 (2016).

45. Li, B. \& Dewey, C. N. RSEM: accurate transcript quantification from RNA-Seq data with or without a reference genome. BMC Bioinformatics 12, 323 (2011).

46. Robinson, M. D., McCarthy, D. J. \& Smyth, G. K. edgeR: a Bioconductor package for differential expression analysis of digital gene expression data. Bioinformatics 26, 139-140 (2010).

47. Kopylova, E., Noé, L. \& Touzet, H. SortMeRNA: fast and accurate filtering of ribosomal RNAs in metatranscriptomic data. Bioinformatics 28, 3211-3217 (2012).

48. Singh, U. \& Wurtele, E. S. orfipy: a fast and flexible tool for extracting ORFs. Bioinformatics btab090 (2021) doi:10.1093/bioinformatics/btab090.

49. Steinegger, M. \& Söding, J. MMseqs2 enables sensitive protein sequence searching for the analysis of massive data sets. Nat Biotechno/ 35, 1026-1028 (2017).

50. NCBI Resource Coordinators et al. Database resources of the National Center for Biotechnology Information. Nucleic Acids Research 46, D8-D13 (2018).

51. Grigoriev, I. V. et al. MycoCosm portal: gearing up for 1000 fungal genomes. Nucl. Acids Res. 42, D699-D704 (2014).

52. Mistry, J. et al. Pfam: The protein families database in 2021. Nucleic Acids Research 49, D412-D419 (2021).

53. Urban, M. et al. PHI-base: the pathogen-host interactions database. Nucleic Acids Research gkz904 (2019) doi:10.1093/nar/gkz904.

54. Sperschneider, J., Dodds, P. N., Gardiner, D. M., Singh, K. B. \& Taylor, J. M. Improved prediction of fungal effector proteins from secretomes with EffectorP 2.0: Prediction of fungal effectors with EffectorP 2.0. Molecular Plant Pathology 19, 2094-2110 (2018).

55. Zhang, H. et al. dbCAN2: a meta server for automated carbohydrate-active enzyme annotation. Nucleic Acids Research 46, W95-W101 (2018).

56. Kanehisa, M., Sato, Y. \& Morishima, K. BlastKOALA and GhostKOALA: KEGG Tools for Functional Characterization of Genome and Metagenome Sequences. Journal of Molecular Biology 428, 726-731 (2016). 
57. Kanehisa, M., Furumichi, M., Sato, Y., Ishiguro-Watanabe, M. \& Tanabe, M. KEGG: integrating viruses and cellular organisms. Nucleic Acids Research 49, D545-D551 (2021).

58. Xu, L. et al. OrthoVenn2: a web server for whole-genome comparison and annotation of orthologous clusters across multiple species. Nucleic Acids Research 47, W52-W58 (2019).

59. Osuna-Cruz, C. M. et al. PRGdb 3.0: a comprehensive platform for prediction and analysis of plant disease resistance genes. Nucleic Acids Research 46, D1197-D1201 (2018).

60. Almagro Armenteros, J. J., Sønderby, C. K., Sønderby, S. K., Nielsen, H. \& Winther, O. DeepLoc: prediction of protein subcellular localization using deep learning. Bioinformatics 33, 3387-3395 (2017).

61. Oono, R., Lefèvre, E., Simha, A. \& Lutzoni, F. A comparison of the community diversity of foliar fungal endophytes between seedling and adult loblolly pines (Pinus taeda). Fungal Biology 119, 917-928 (2015).

62. Würth, D. G. et al. The needle mycobiome of Picea glauca - A dynamic system reflecting surrounding environment and tree phenological traits. Fungal Ecology 41, 177-186 (2019).

63. Arnold, A. E. Understanding the diversity of foliar endophytic fungi: progress, challenges, and frontiers. Fungal Biology Reviews 21, 51-66 (2007).

64. Bass, D., Stentiford, G. D., Wang, H.-C., Koskella, B. \& Tyler, C. R. The Pathobiome in Animal and Plant Diseases. Trends in Ecology \& Evolution 34, 996-1008 (2019).

65. Hunt, R. S., Ying, C. C. \& Ashbee, D. Variation in damage among Pinus contorta provenances caused by the needle cast fungus Lophodermella concolor. Canadian Journal of Forest Research 17, 594-597 (1987).

66. Monack, D. M., Mueller, A. \& Falkow, S. Persistent bacterial infections: the interface of the pathogen and the host immune system. Nat Rev Microbio/ 2, 747-765 (2004).

67. Pham, T. A. N. \& Lawley, T. D. Emerging insights on intestinal dysbiosis during bacterial infections. Current Opinion in Microbiology 17, 67-74 (2014).

68. Petersen, C. \& Round, J. L. Defining dysbiosis and its influence on host immunity and disease. Cell Microbio/ 16, 1024-1033 (2014).

69. Liu, H., Brettell, L. E. \& Singh, B. Linking the Phyllosphere Microbiome to Plant Health. Trends in Plant Science 25, 841-844 (2020).

70. Jenior, M. L., Leslie, J. L., Young, V. B. \& Schloss, P. D. Clostridium difficile Alters the Structure and Metabolism of Distinct Cecal Microbiomes during Initial Infection To Promote Sustained Colonization. $m S p h e r e<$ bverticalalign:super;>3</bvertical-align:super;>, e00261-18, /msphere/3/3/mSphere261-18.atom (2018).

71. Sorbara, M. T. \& Pamer, E. G. Interbacterial mechanisms of colonization resistance and the strategies pathogens use to overcome them. Mucosal Immunol 12, 1-9 (2019).

72. Pickard, J. M., Zeng, M. Y., Caruso, R. \& Núñez, G. Gut microbiota: Role in pathogen colonization, immune responses, and inflammatory disease. Immunol Rev 279, 70-89 (2017).

73. O'Connell, R. J. et al. Lifestyle transitions in plant pathogenic Colletotrichum fungi deciphered by genome and transcriptome analyses. Nat Genet 44, 1060-1065 (2012).

74. Teixeira, P. J. P. L. et al. High-Resolution Transcript Profiling of the Atypical Biotrophic Interaction between Theobroma cacao and the Fungal Pathogen Moniliophthora perniciosa. The Plant Cell 26, 4245-4269 (2014).

75. Deckert, R. J., Hsiang, T. \& Peterson, R. L. Genetic relationships of endophytic Lophodermium nitens isolates from needles of Pinus strobus. Mycological Research 106, 305-313 (2002).

76. Stone, J. K. Initiation and development of latent infections by Rhabdocline parkeri on Douglas-fir. Can. J. Bot. 65, 2614-2621 (1987). 
77. Yang, F., Li, W. \& Jørgensen, H. J. L. Transcriptional Reprogramming of Wheat and the Hemibiotrophic Pathogen Septoria tritici during Two Phases of the Compatible Interaction. PLoS ONE 8, e81606 (2013).

78. Ye, W. et al. Disclosure of the Molecular Mechanism of Wheat Leaf Spot Disease Caused by Bipolaris sorokiniana through Comparative Transcriptome and Metabolomics Analysis. IJMS 20, 6090 (2019).

79. Vleeshouwers, V. G. A. A. \& Oliver, R. P. Effectors as Tools in Disease Resistance Breeding Against Biotrophic, Hemibiotrophic, and Necrotrophic Plant Pathogens. MPMI 27, 196-206 (2014).

80. Solomon, P. Have we finally opened the door to understanding Septoria tritici blotch disease in wheat? The New Phytologist 214, 493-495 (2017).

81. Howlett, B. J. Secondary metabolite toxins and nutrition of plant pathogenic fungi. Current Opinion in Plant Biology 9, 371-375 (2006).

82. Abou Ammar, G. et al. Identification of ABC Transporter Genes of Fusarium graminearum with Roles in Azole Tolerance and/or Virulence. PLOS ONE 8, e79042 (2013).

83. Perlin, M. H., Andrews, J. \& San Toh, S. Essential Letters in the Fungal Alphabet. in Advances in Genetics vol. 85 201-253 (Elsevier, 2014).

84. Zwiers, L.-H., Stergiopoulos, I., Gielkens, M. M. C., Goodall, S. D. \& De Waard, M. A. ABC transporters of the wheat pathogen Mycosphaerella graminicola function as protectants against biotic and xenobiotic toxic compounds. Mol Gen Genomics 269, 499-507 (2003).

85. Eaton, C. J., Cox, M. P. \& Scott, B. What triggers grass endophytes to switch from mutualism to pathogenism? Plant Science 180, 190-195 (2011).

86. Tang, D., Wang, G. \& Zhou, J.-M. Receptor Kinases in Plant-Pathogen Interactions: More Than Pattern Recognition. Plant Cell 29, 618-637 (2017).

87. Afzal, A. J., Wood, A. J. \& Lightfoot, D. A. Plant Receptor-Like Serine Threonine Kinases: Roles in Signaling and Plant Defense. MPMI<bvertical-align:super;>21</bvertical-align:super;>, 507-517 (2008).

88. Jagodzik, P., Tajdel-Zielinska, M., Ciesla, A., Marczak, M. \& Ludwikow, A. Mitogen-Activated Protein Kinase Cascades in Plant Hormone Signaling. Front. Plant Sci. 9, 1387 (2018).

89. Zhao, J. Phospholipase D and phosphatidic acid in plant defence response: from protein-protein and lipidprotein interactions to hormone signalling. Journal of Experimental Botany 66, 1721-1736 (2015).

90. Sun, X. et al. Functional characterization of an Arabidopsis prolyl aminopeptidase AtPAP1 in response to salt and drought stresses. Plant Cell Tiss Organ Cult 114, 325-338 (2013).

91. Szawłowska, U., Grabowska, A., Zdunek-Zastocka, E. \& Bielawski, W. TsPAP1 encodes a novel plant prolyl aminopeptidase whose expression is induced in response to suboptimal growth conditions. Biochemical and Biophysical Research Communications 419, 104-109 (2012).

92. Wang, Y., Liu, H., Wang, S., Li, H. \& Xin, Q. Overexpressing of a novel wheat prolyl aminopeptidase gene enhances zinc stress tolerance in transgenic Arabidopsis thaliana. Plant Cell Tiss Organ Cult 121, 489-499 (2015).

93. Nixon, P. J. FtsH-mediated repair of the photosystem II complex in response to light stress. Journal of Experimental Botany 56, 357-363 (2004).

94. Minic, Z. Physiological roles of plant glycoside hydrolases. Planta 227, 723-740 (2008).

95. Minic, Z. \& Jouanin, L. Plant glycoside hydrolases involved in cell wall polysaccharide degradation. Plant Physiology and Biochemistry 44, 435-449 (2006).

96. Bruggeman, Q. et al. The Polyadenylation Factor Subunit CLEAVAGE AND POLYADENYLATION SPECIFICITY FACTOR30: A Key Factor of Programmed Cell Death and a Regulator of Immunity in Arabidopsis. Plant 
Physiology 165, 732-746 (2014).

97. Hopkins, M. T., Lampi, Y., Wang, T.-W., Liu, Z. \& Thompson, J. E. Eukaryotic Translation Initiation Factor 5A Is Involved in Pathogen-Induced Cell Death and Development of Disease Symptoms in Arabidopsis. Plant Physiol. 148, 479-489 (2008).

98. Ruan, B. et al. Os ACL -A2 negatively regulates cell death and disease resistance in rice. Plant Biotechnol J 17, 1344-1356 (2019).

99. País, S. M., Téllez-Iñón, M. T. \& Capiati, D. A. Serine/Threonine Protein Phosphatases type 2A and their roles in stress signaling. Plant Signaling \& Behavior 4, 1013-1015 (2009).

100. Mahesh, S. K., Liu, H. \& Qiu, D. The Role of Radical Burst in Plant Defense Responses to Necrotrophic Fungi. Journal of Integrative Agriculture 11, 1305-1312 (2012).

101. Shetty, N. P. et al. Role of hydrogen peroxide during the interaction between the hemibiotrophic fungal pathogen Septoria tritici and wheat. New Phytologist 174, 637-647 (2007).

102. Ali, S. S. et al. Phytophthora megakarya and Phytophthora palmivora, Closely Related Causal Agents of Cacao Black Pod Rot, Underwent Increases in Genome Sizes and Gene Numbers by Different Mechanisms. Genome Biology and Evolution 9, 536-557 (2017).

103. Belibasakis, G. N. \& Manoil, D. Microbial Community-Driven Etiopathogenesis of Peri-Implantitis. J Dent Res 100, 21-28 (2021).

104. Kashyap, P. L., Rai, P., Kumar, S., Chakdar, H. \& Srivastava, A. K. DNA Barcoding for Diagnosis and Monitoring of Fungal Plant Pathogens. in Molecular Markers in Mycology (eds. Singh, B. P. \& Gupta, V. K.) 87-122 (Springer International Publishing, 2017). doi:10.1007/978-3-319-34106-4_5.

105. Martinez, K. B., Leone, V. \& Chang, E. B. Microbial metabolites in health and disease: Navigating the unknown in search of function. Journal of Biological Chemistry 292, 8553-8559 (2017).

106. Ahrendt, S. R. et al. Leveraging single-cell genomics to expand the fungal tree of life. Nat Microbio/3, 1417-1428 (2018).

107. Magan, N. \& Smith, M. K. Isolation of the endophytes Lophodermium piceae and Rhizosphaera kalkhoffii from Sitka spruce needles in poor and good growth sites and in vitro effects of environmental factors. Phyton 36, 103-110 (1996).

108. Kowalski, T. \& Krygier, J. Mycological study on symptomless and diseased needles in pine stand attacked by Lophodermella sulcigena (Rostr.) V. Höhn. Phytopathologia Polonica 159-168 (1996).

109. Ryan, E. T. The Intestinal Pathobiome: Its Reality and Consequences Among Infants and Young Children in Resource-Limited Settings. Journal of Infectious Diseases 208, 1732-1733 (2013).

110. Schulz, B. \& Boyle, C. The endophytic continuum. Mycological Research 109, 661-686 (2005).

111. Précigout, P.-A., Claessen, D., Makowski, D. \& Robert, C. Does the Latent Period of Leaf Fungal Pathogens Reflect Their Trophic Type? A Meta-Analysis of Biotrophs, Hemibiotrophs, and Necrotrophs. Phytopathology ${ }^{\circledR}<$ bverticalalign:super;>110</bvertical-align:super;>, 345-361 (2020).

\section{Figures}




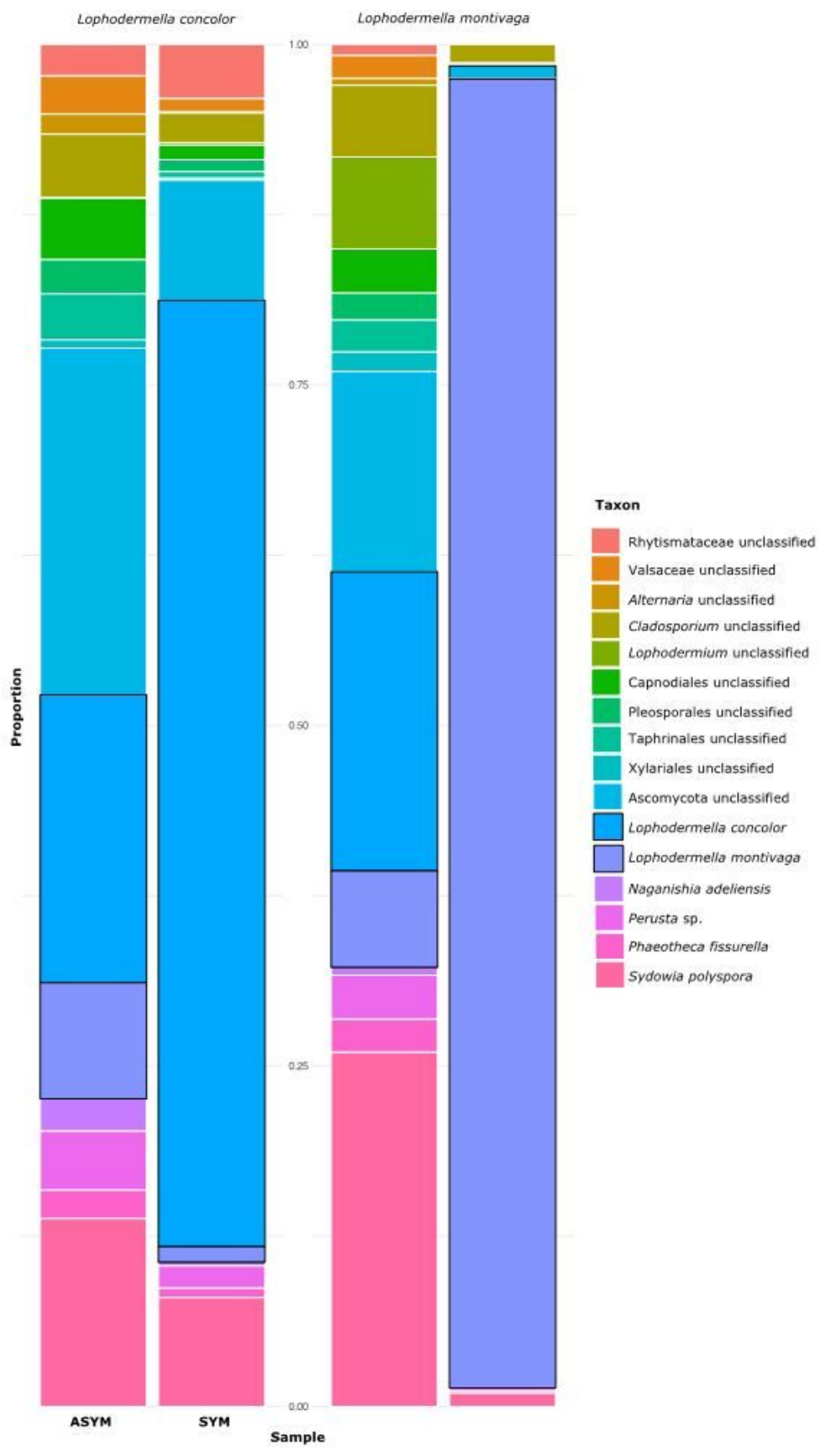

Figure 1

Relative abundance of fungal taxa within the mycobiome across Pinus contorta needles that were asymptomatic (ASYM) and symptomatic (SYM) of Lophodermella concolor and L. montivaga identified through metabarcoding. 


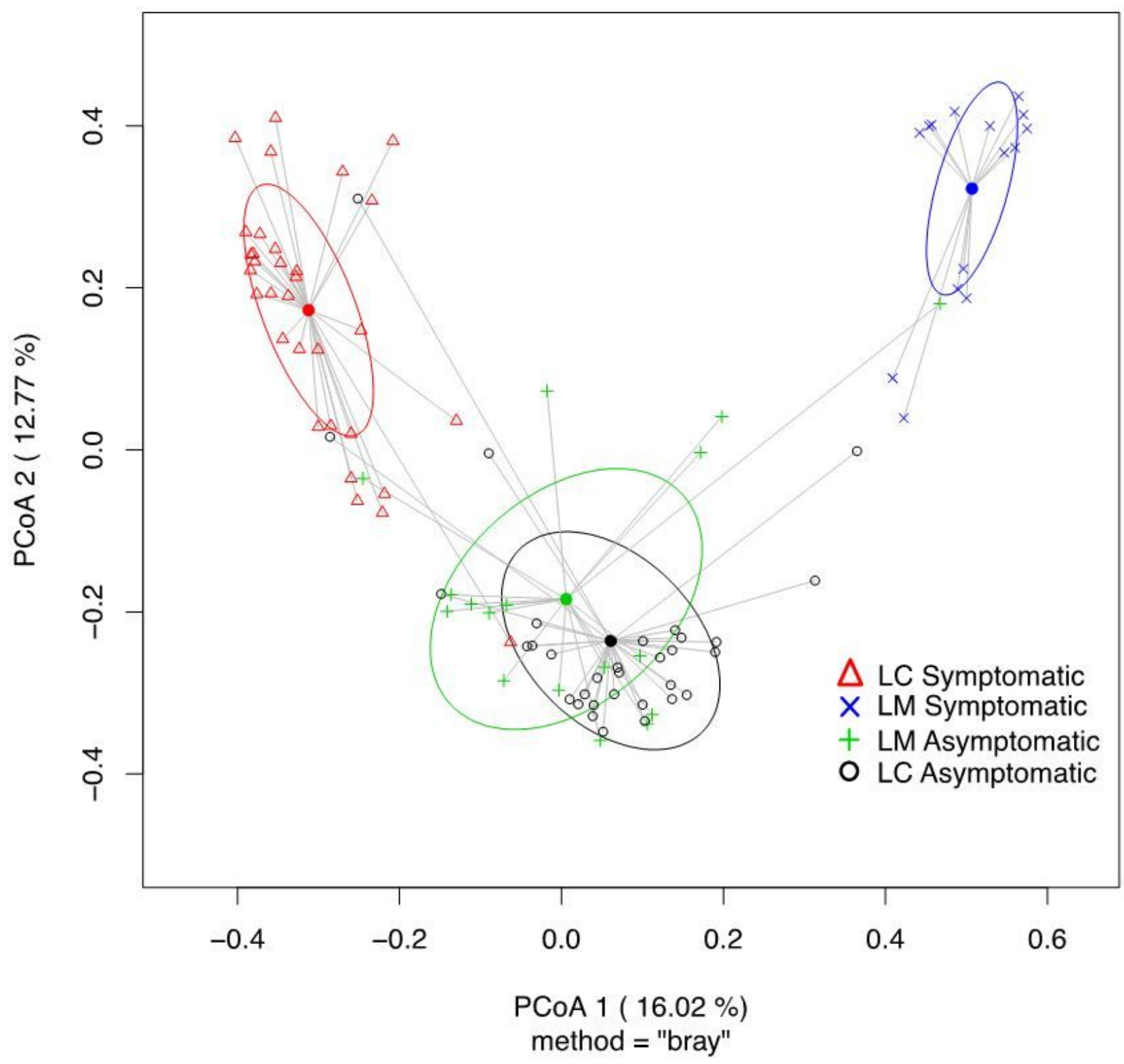

Figure 2

Principal coordinate analysis (PCOA) based on relative abundance of fungal operational taxonomic units (OTUs) showing fungal community structure on Pinus contorta needles that are symptomatic or symptomatic of Lophodermella concolor (LC) and L. montivaga (LM). Ellipses represent one standard deviation. 


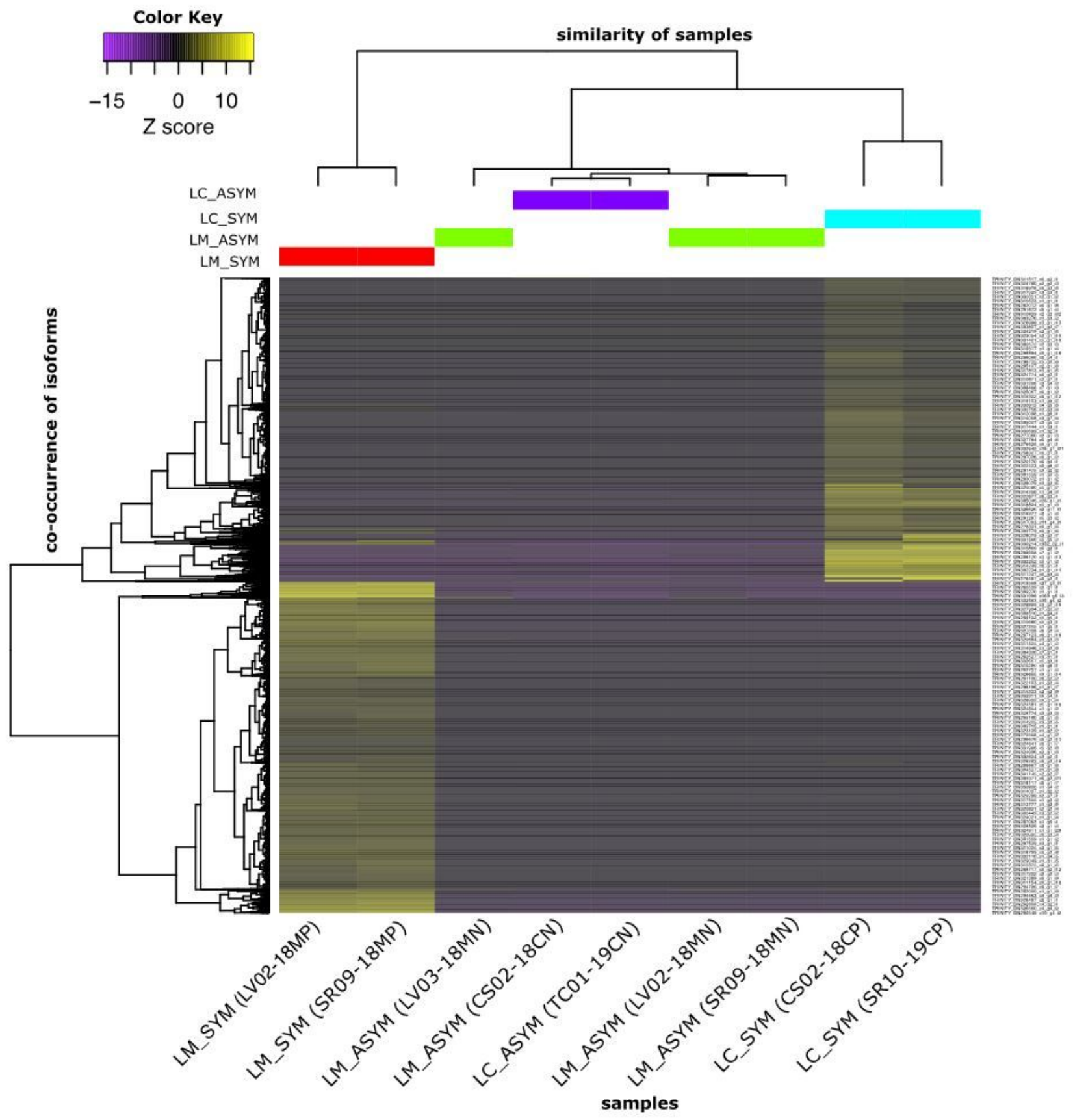

\section{Figure 3}

Heatmap of the differentially expressed transcripts (isoforms) in P. contorta needles that were asymptomatic (ASYM) and symptomatic (SYM) of Lophodermella concolor (LC) or L. montivaga (LM) based on counts per million (CPM) reads. Trinity transcripts shown are differentially expressed features ( $p$-value $<0.05$, fold change ${ }^{3} 2$ ) within each of the four pairwise comparisons. Similar colors at tree tips represent replicate samples within each of the following treatments: LC_ASYM (purple), LC_SYM (blue), LM_ASYM (green), and LM_SYM (red). 


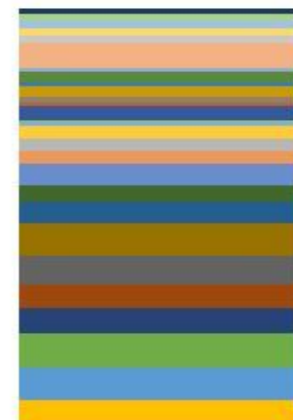

\section{Family}

- Hypocreaceae

॥erpotrichiellaceae

॥Plectosphaerellaceae

7000

unclassified Helotiales

- Clavicipitaceae

- Pseudeurotiaceae

-Valsaceae

- Chlorociboriaceae

- Pleuroascaceae

-Erysiphaceae

- Drepanopezizaceae

-Hypoxylaceae

- Leotiomycetes incertae sedis

- Ophiocordycipitaceae

-Botryosphaeriaceae

॥Nectriaceae

- Glomerellaceae

॥ Rutstroemiaceae

- Aspergillaceae

- Helotiaceae

-Myxotrichaceae

-Mollisiaceae

-Dermateaceae

- Pseudeurotiaceae

- Helotiales incertae sedis

- Sclerotiniaceae

- Hyaloscyphaceae

- Lachnaceae

- Cudoniaceae

- Triblidiaceae

- Rhytismataceae

1000

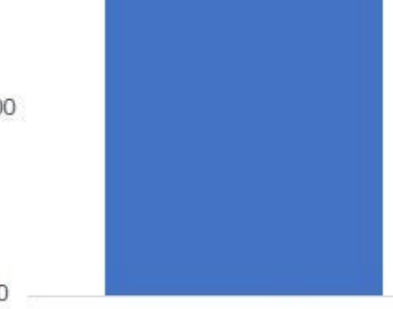

LC_SYM

\section{Figure 4}

Abundance of the top 25 taxonomic families of differentially expressed (DE) fungal transcripts among $P$. contorta needles symptomatic (SYM) of Lophodermella concolor (LC) or L. montivaga (LM) when compared to their corresponding asymptomatic needles (LC_ASYM vs. LC_SYM and LM_ASYM vs. LM_SYM); in these two comparisons, no fungal DE transcripts in asymptomatic needles were among the top 25 families. Taxonomic annotations were obtained from concatenated databases of NCBI-nr and JGI Mycocosm. 

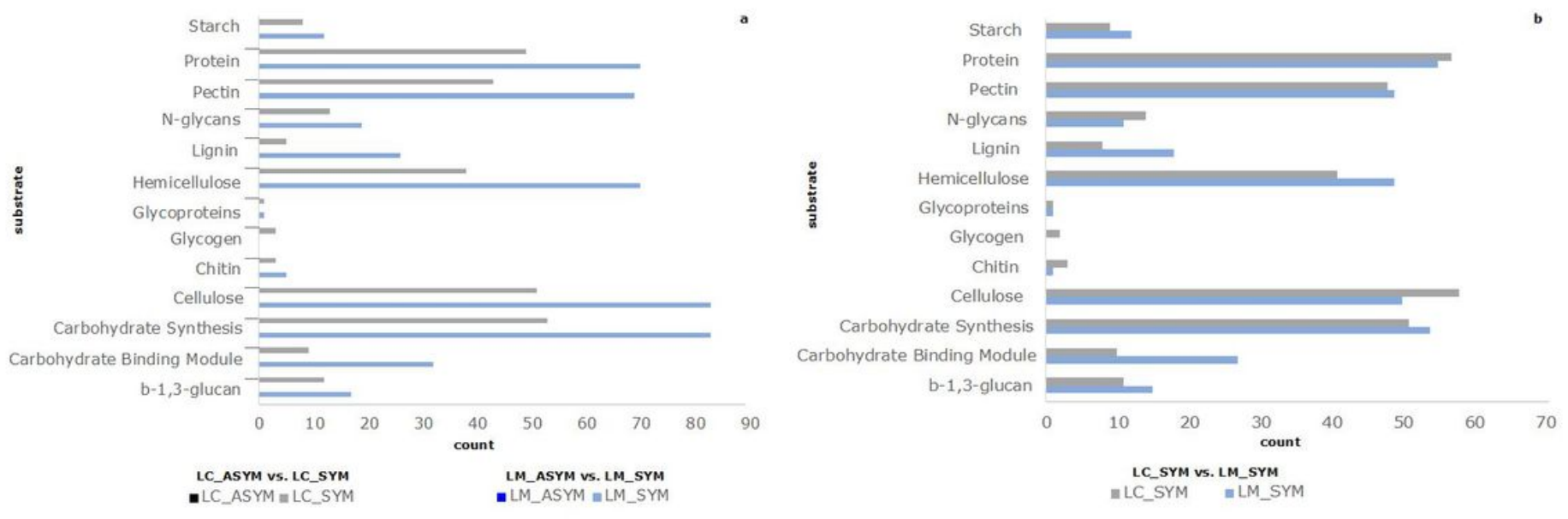

\section{Figure 5}

Number of fungal enzymes that degrade proteins and various substrates that were differentially expressed between (a) Pinus contorta needles symptomatic and asymptomatic of Lophodermella concolor(LC_ASYM vs. LC_SYM) and L. montivaga (LM_ASYM vs. LM_SYM), and (b) between symptomatic needles of L. concolor and L. montivaga (LC_SYM vs. LM_SYM) inferred from dbCAN2 and PFAM.
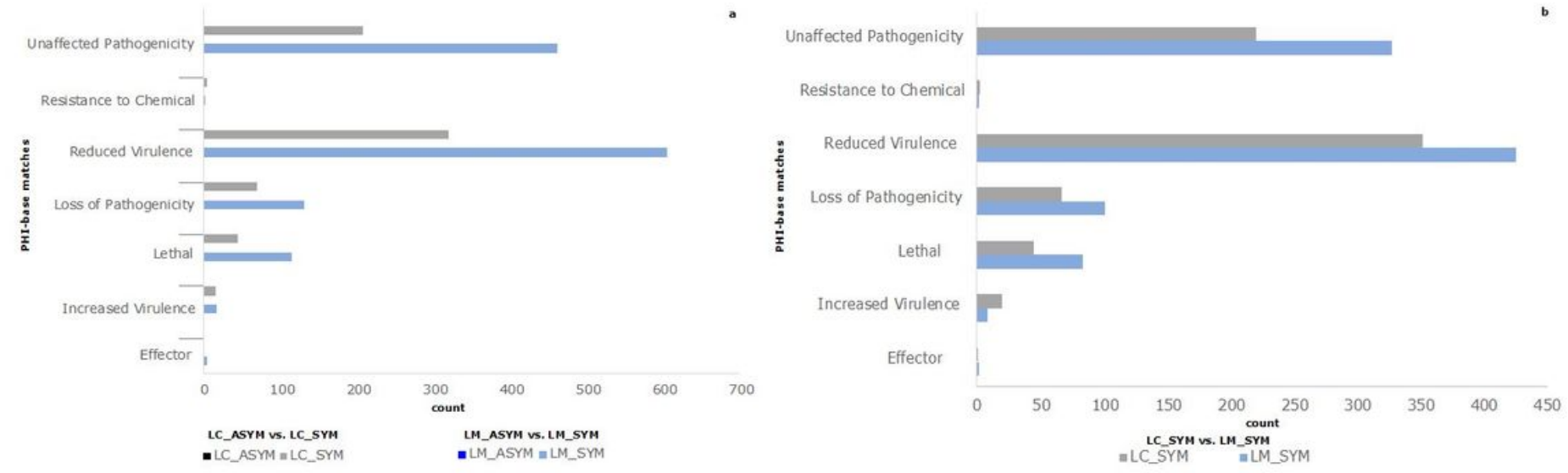

\section{Figure 6}

PHI-base hits of fungal transcripts between (a) Pinus contorta needles symptomatic and asymptomatic of Lophodermella concolor (LC_ASYM vs. LC_SYM) and L. montivaga (LM_ASYM vs. LM_SYM), and (b) between symptomatic needles of L. concolor and L. montivaga (LC_SYM vs. LM_SYM). 

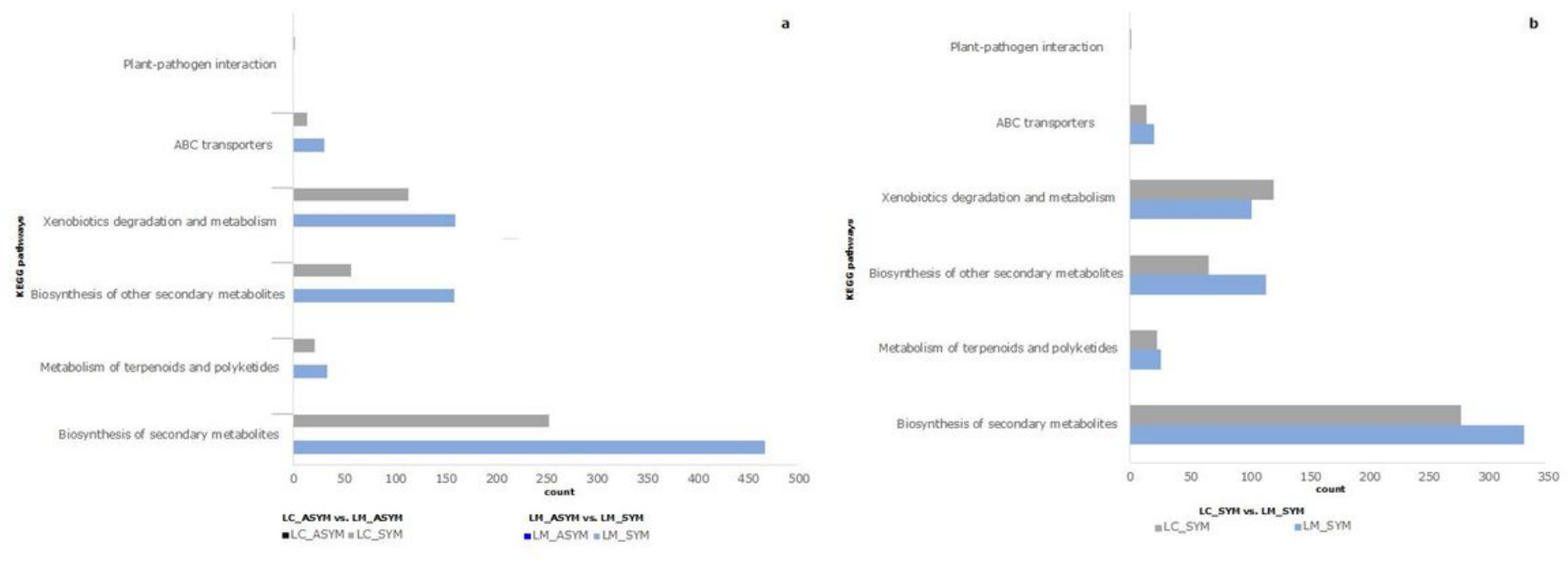

Figure 7

Number of fungal transcripts involved in the production of secondary metabolites, environmental adaptation, and membrane transport between (a) Pinus contorta needles symptomatic and asymptomatic of Lophodermella concolor (LC_ASYM vs. LC_SYM) and L. montivaga (LM_ASYM vs. LM_SYM), and (b) between symptomatic needles of $L$. concolor and L. montivaga (LC_SYM vs. LM_SYM) inferred from KEGG.

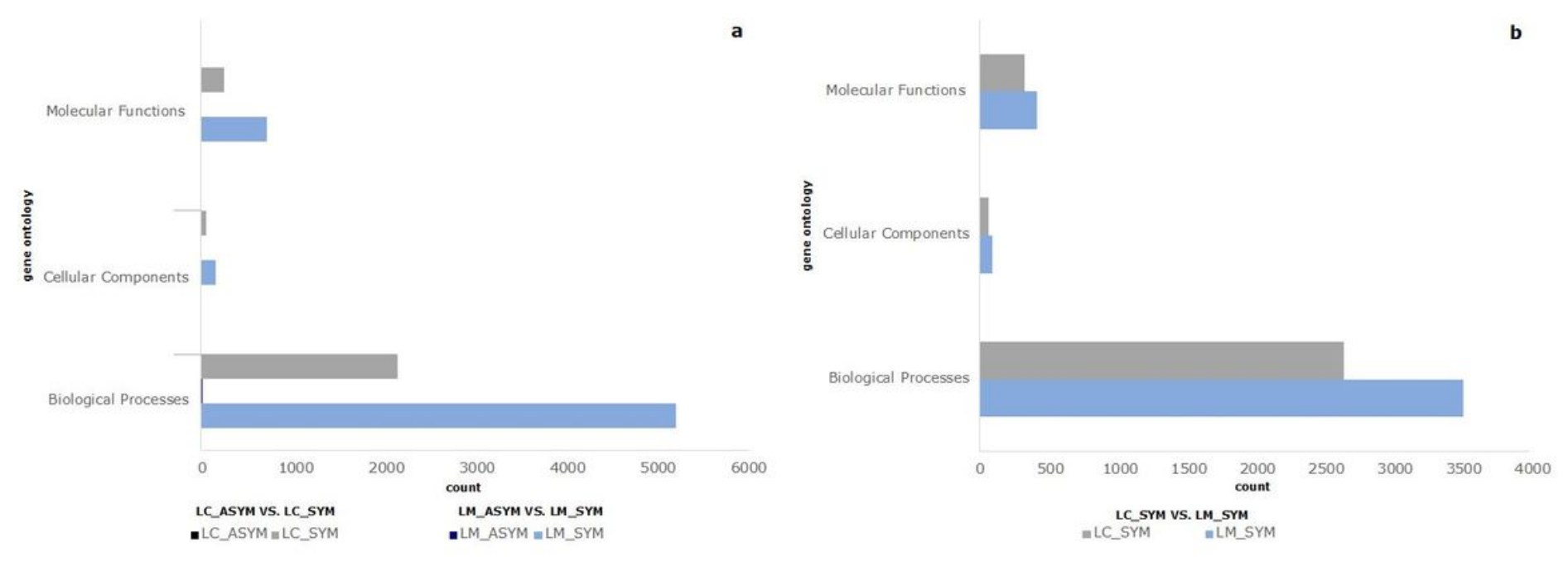

\section{Figure 8}

Gene ontologies of fungal DE transcripts between (a) Pinus contorta needles symptomatic and asymptomatic of Lophodermella concolor(LC_ASYM vs. LC_SYM) and L. montivaga (LM_ASYM vs. LM_SYM), and (b) between symptomatic needles of L. concolor and L. montivaga (LC_SYM vs. LM_SYM) inferred from Orthovenn2. 

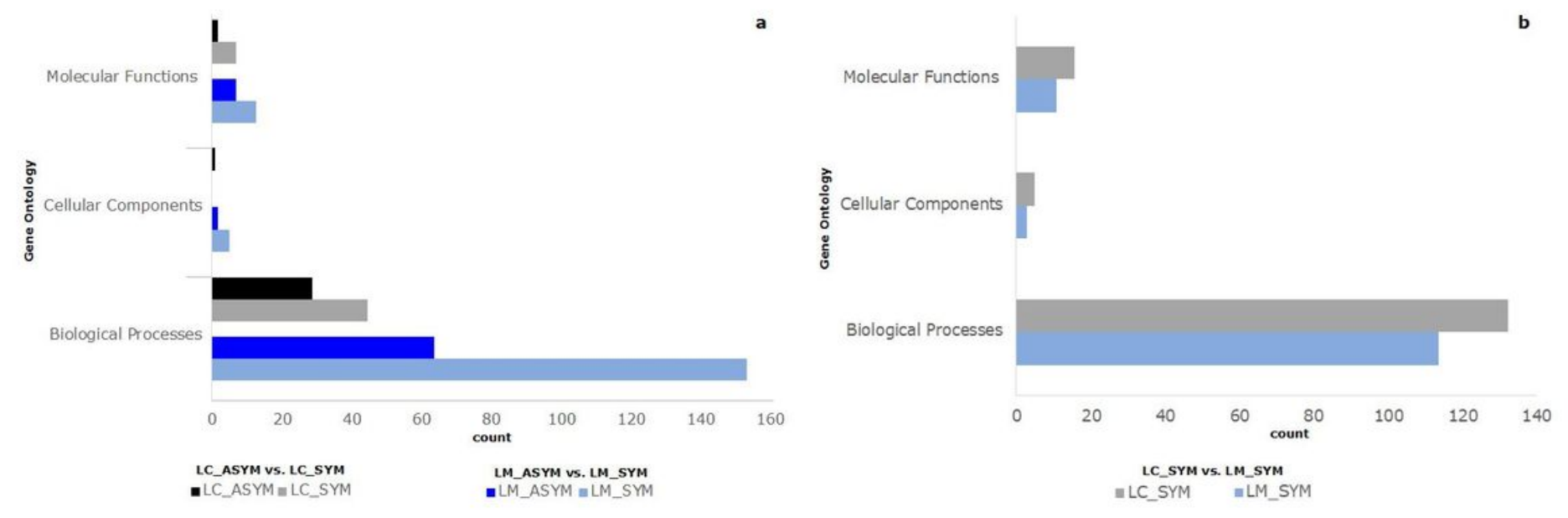

Figure 9

Gene ontologies of plant DE transcripts between (a) Pinus contorta needles symptomatic and asymptomatic of Lophodermella concolor(LC_ASYM vs. LC_SYM) and L. montivaga (LM_ASYM vs. LM_SYM), and (b) between symptomatic needles of L. concolor and L. montivaga (LC_SYM vs. LM_SYM) inferred from Orthovenn2.

\section{Supplementary Files}

This is a list of supplementary files associated with this preprint. Click to download.

- AtaetalSupplementarytables.xlsx

- AtaetalSupportingInformation5Nov2021.docx 